\title{
Plasma-assisted discharges and charging in EUV-induced plasma
}

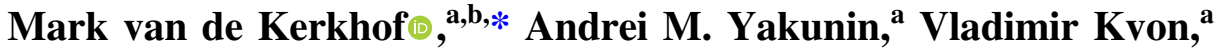 \\ Selwyn Cats, ${ }^{a}$ Luuk Heijmans, ${ }^{a}$ Manis Chaudhuri, ${ }^{a}$ and Dmitry Asthakov ${ }^{c, d}$ \\ ${ }^{a}$ ASML Netherlands B.V., Veldhoven, The Netherlands \\ ${ }^{b}$ Eindhoven University of Technology, Department of Applied Physics, Eindhoven, \\ The Netherlands \\ 'ISTEQ B.V., Eindhoven, The Netherlands \\ ${ }^{\mathrm{d}}$ Institute for Spectroscopy of the Russian Academy of Sciences, Moscow, Russia
}

\begin{abstract}
In the past years, EUV lithography scanner systems have entered high-volume manufacturing for state-of-the-art integrated circuits (IC), with critical dimensions down to $10 \mathrm{~nm}$. This technology uses 13.5-nm EUV radiation, which is transmitted through a near-vacuum $\mathrm{H}_{2}$ background gas, imaging the pattern of a reticle onto a wafer. The energetic EUV photons excite the background gas into a low-density $\mathrm{H}_{2}$ plasma. The resulting plasma will locally change the near-vacuum into a conducting medium and can charge floating surfaces and particles, also away from the direct EUV beam. We will discuss the interaction between EUV-induced plasma and electrostatics, by modeling and experiments. We show that the EUV-induced plasma can trigger discharges well below the classical Paschen limit. Furthermore, we demonstrate the charging effect of the EUV plasma on both particles and surfaces. Uncontrolled, this can lead to unacceptably high voltages on the reticle backside and the generation and transport of particles. We demonstrate a special unloading sequence to use the EUV-induced plasma to actively solve the charging and defectivity challenges. () The Authors. Published by SPIE under a Creative Commons Attribution 4.0 Unported License. Distribution or reproduction of this work in whole or in part requires full attribution of the original publication, including its DOI. [DOI: 10.1117/1.JMM.20.1.013801]
\end{abstract}

Keywords: EUV lithography; EUV-induced plasma; reticle; particles; electrostatics; discharge.

Paper 20053 received Nov. 30, 2020; accepted for publication Jan. 29, 2021; published online Feb. 19, 2021.

\section{Introduction}

The ongoing technological evolution in integrated circuits (IC's) is driven by an exponential growth in demand for computing power and data transport and is expected to accelerate further in coming years with the advent of artificial intelligence running partly on high-performance centralized servers but also on local and mobile edge-computing devices. Power consumption and computing performance will be key drivers for improving architectures as well as further increases in pattern density. Driven by Moore's law, ${ }^{1}$ named after Intel cofounder Gordon Moore, the critical dimensions of IC's have shrunk by a factor of 2 every 1.5 to 2 years; nowadays, the critical dimensions of the most advanced devices are in order of $10 \mathrm{~nm}$. This has been enabled by advances in all processing steps, but mainly by continuous advances in photolithography, by decreasing the (UV) wavelength and increasing the numerical aperture of the photolithographic tools (also known as scanners, see Fig. 1), and introducing resolution enhancements such as polarization ${ }^{2,3}$ and immersion. ${ }^{4}$ Recently, the introduction of extreme ultraviolet (EUV) scanners into high-volume manufacturing ${ }^{5}$ has ensured that Moore's law can continue for the coming years. ${ }^{6}$

Even with the outstanding imaging and overlay capability of the EUV scanners, device yield can still be affected adversely by other factors, such as particles ending up on critical surfaces. The current state-of-the-art lithography node has critical device structures of below $20 \mathrm{~nm}$ at wafer level. This translates to $80 \mathrm{~nm}$ at reticle level (multiplied by $4 \times$, because of the scanner

*Address all correspondence to Mark van de Kerkhof, mark.van.de.kerkhof-msd@asml.com 


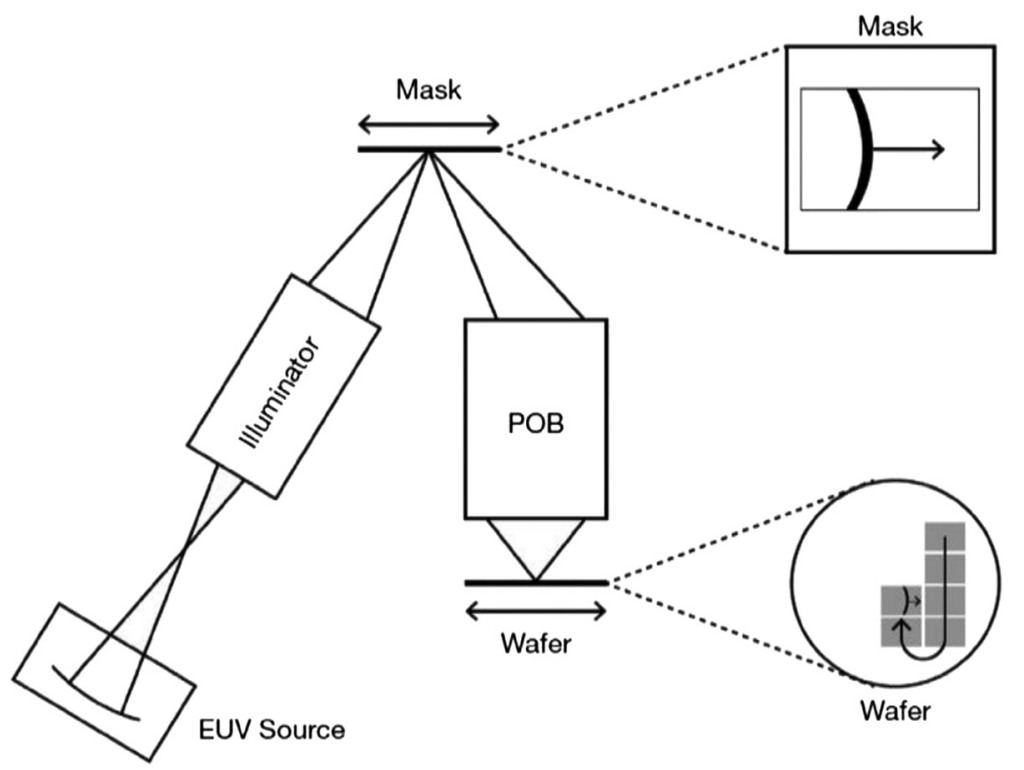

Fig. 1 Basic principle of an EUV scanner; the object on a reticle (or mask) is illuminated and imaged onto a portion of a wafer by the projection optics box (POB) while scanning; after which the wafer is moved to a new position and the process is repeated (source: ASML).

demagnification $M=1 / 4$ ), and a printing particle size limit of $d_{c}=\sim 40 \mathrm{~nm}$ at reticle level. For future nodes, this printing defect size will decrease further. Therefore, particle contamination control, or defectivity control, is a key aspect of scanner system design. The industry is pursuing a dual-path approach to defectivity. One path is advanced particle contamination control to ensure zero particles reaching the sensitive reticle and wafer surfaces. In parallel, EUVcompatible pellicles are now available (transparent films shielding the reticle from particles), which are fully supported by the scanner. ${ }^{7}$ Pellicles have intrinsic benefits in terms of particles but come at the cost of reduced system transmission and productivity; the cost/risk-driven trade-off between the two options depends on application details and can vary for different chip manufacturers and even for different exposure layers. ${ }^{8}$ This paper will focus on the case of advanced particle contamination control, without pellicle.

Particles can come from parts and scanner integration or can be generated by the moving parts in the scanner or can be carried in with the reticles and wafers. It was found that the EUVinduced plasma in the scanner can be a major factor in releasing and transporting particles via electrostatic release and underetching. ${ }^{9}$ This plasma is the result of ionization of the protective hydrogen background gas in the scanner. ${ }^{10}$ Besides direct impact on particles, the EUV-induced plasma can also interact with electrostatics in several ways: it can, e.g., reduce the safe voltage in terms of gas breakdown. In the subsequent sections, these aspects will be described in more detail and design consideration will be discussed.

\section{EUV-Induced Plasma}

Current EUV sources for lithography are based on EUV emission by a hot Sn (tin) plasma: this Sn plasma is formed by irradiating a stream of Sn droplets by a high-power pulsed IR laser (laser-produced plasma, LPP). ${ }^{11}$ The Sn plasma has a peak temperature of several tens of $\mathrm{eV}$, which efficiently emits EUV radiation around $13.5 \mathrm{~nm}$. High conversion efficiency is achieved by an IR prepulse to enlarge the target and reduce the Sn density. ${ }^{12}$ The raw emission spectrum is broadband and includes longer-wavelength vacuum UV components, but in the scanner this spectrum is filtered by the narrow-band Bragg-reflection mirrors to $13.5 \mathrm{~nm}$ $( \pm 0.2 \mathrm{~nm}) .{ }^{13}$ Current LPP EUV sources are highly transient, firing short $<100 \mathrm{~ns}$ pulses with energy of $\sim 5 \mathrm{~mJ}$ at $50 \mathrm{kHz}$ ( $250 \mathrm{~W}$ output), with the peak of EUV in the first $50 \mathrm{~ns}$ and a tail of broadband radiation. 


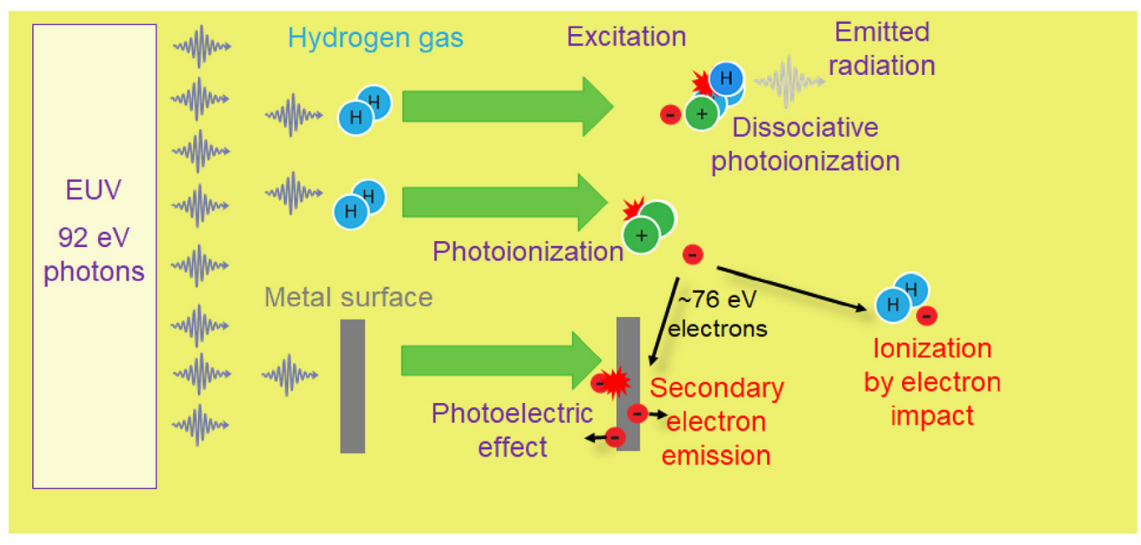

Fig. 2 Basic processes of EUV-induced hydrogen plasma.

EUV lithography employs a low-pressure background gas of 1 to $10 \mathrm{~Pa}$ hydrogen $\left(\mathrm{H}_{2}\right)$, to maintain self-cleaning conditions for the sensitive EUV mirrors in the optical system of the scanner. Hydrogen was chosen as background gas, because of high chemical activity of H-ions, ${ }^{14}$ high transmission of $\mathrm{H}_{2}$ for EUV, and low/negligible sputtering by the light $\mathrm{H}$-ions. The 92-eV EUV photons will lead to some absorption and photo-ionization of the hydrogen background gas, creating a plasma, as outlined in Fig. 2. Because of the low absorption of $\mathrm{H}_{2}$ (attenuation coefficient $\alpha=0.0078 \mathrm{~m}^{-1}$ at $5 \mathrm{~Pa}$ ), the ionization degree will be low at $\sim 10^{-4} \%$ (for a $250-\mathrm{W}$ source), and secondary interactions between hot electrons and ions will have low probability. Momentum conservation dictates that the large excess energy of $76 \mathrm{eV}$ is carried by the photoelectron while the ions remain at room temperature. ${ }^{15}$ These hot photoelectrons will lose energy by secondary ionizations and dissociations of the neutral hydrogen molecules within first $2 \mu$ s; this results in an increase of plasma density even after the EUV pulse has passed and to formation of up to three pairs of ions and electrons per absorbed photon. ${ }^{16}$

When the electron energy is reduced to beneath $20 \mathrm{eV}$ by these secondary events, subsequent cooling will slow down and the plasma will decay by diffusion and wall recombination. In view of the low ionization degree and the proximity of wall surfaces, volume recombination will be a minor effect and can be effectively ignored. ${ }^{17}$ During the EUV pulse of $<100 \mathrm{~ns}$, the plasma will instantaneously expand several centimeter's beyond the EUV beam by the fast photoelectrons and subsequently expand and decay by diffusion. Given the LPP source frequency of $50 \mathrm{kHz}$, this cycle repeats every $20 \mu \mathrm{s}$; as the decay time of the plasma will typically exceed $20 \mu \mathrm{s}$ at pressures of 1 to $10 \mathrm{~Pa},{ }^{18}$ there will be build-up of a steady-state plasma, with repeating highly transient peaks every $20 \mu \mathrm{s}$, as shown in Fig. 3. The resulting quasi-steady-state background

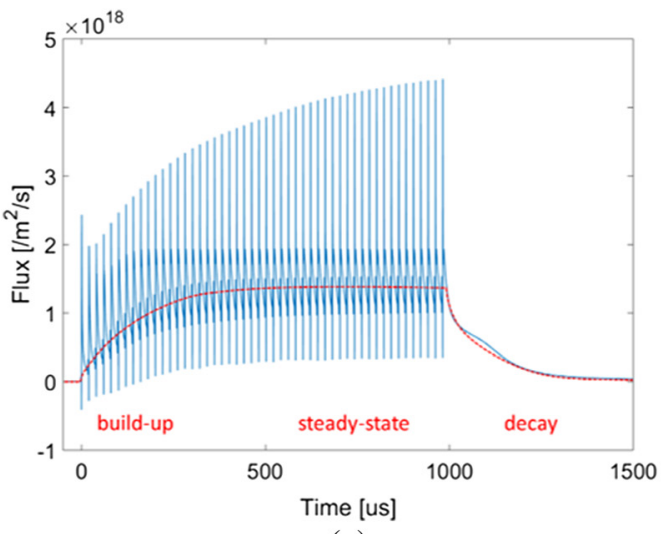

(a)

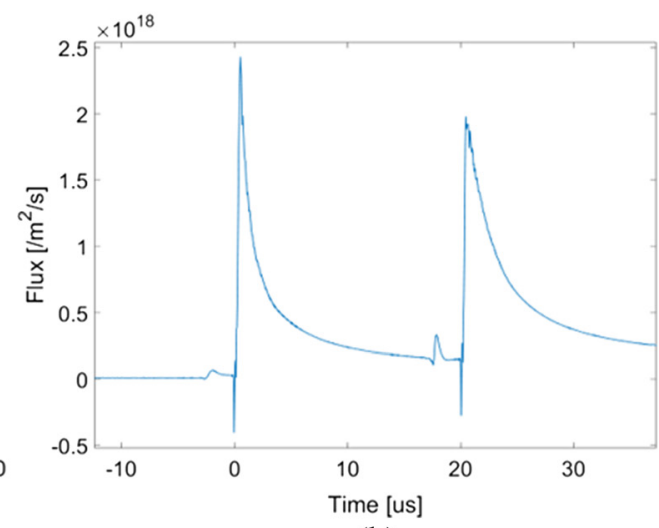

(b)

Fig. 3 (a) Build-up, steady-state, and decay of pulsed EUV-induced plasma, as measured by retarding field energy analyzer in scanner-like test-stand directly after LPP source exit. ${ }^{19}$ (b) Zoom-in on first two pulses; also visible is the minor plasma formation due to the prepulse. 


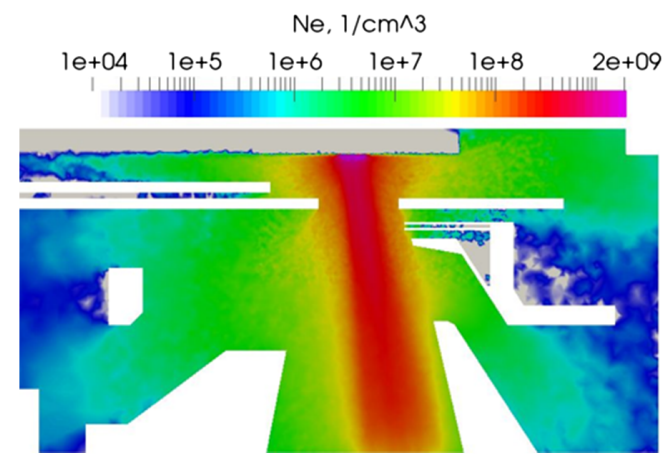

(a)

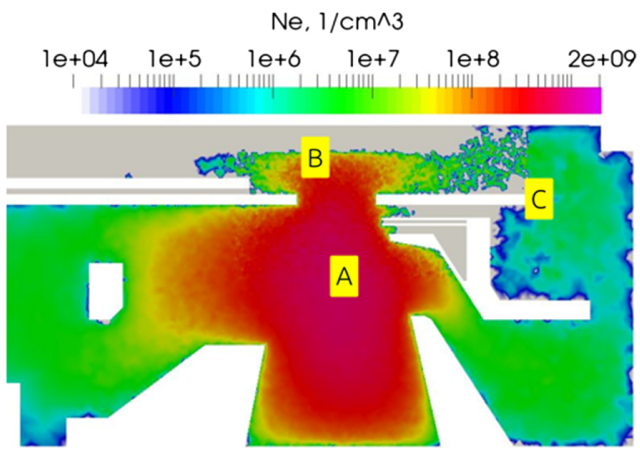

(b)

Fig. 4 Schematic of reticle zone, showing (A) EUV beam region, (B) floating reticle surface, and (C) grounded reticle masking blades. (a) During isolated EUV pulse the electrons from the EUV beam penetrate through the slits. (b) Plasma accumulation beyond the beam confines over multiple pulses.

plasma can be described as weakly coupled, diffuse and cold; but the transient plasma will typically not be in local thermal equilibrium, and the electron energy distribution will not be Maxwellian. ${ }^{20}$ This in turn means that many classical plasma assumptions will not or not always apply, and care must be taken with classical equations for plasma temperature, Debye shielding length, and plasma sheath.

The energetic photoelectrons will set up a significant plasma-to-wall potential difference in order of $76 \mathrm{~V}$, peaking during the EUV pulse and dropping fast in the afterglow. ${ }^{21}$ In practice, this plasma-wall potential will be reduced by photoelectric effect, which results in low-energy electrons being released from any wall (or mirror) irradiated by the EUV beam or by EUV flare, ${ }^{15}$ by secondary electron emission, and by charge compensation by ions; the resulting steady state potential of floating surfaces and dielectrics exposed to the EUV-induced plasma is in order of a few volts (typically $\sim 2 \mathrm{~V}$ ). As the mean free path of the energetic photoelectrons can be several centimeters (given electron-neutral collision cross-section $\sigma$ of $\sim 10^{-20} \mathrm{~m}^{2}$ at $\sim 76 \mathrm{eV}$; from Ref. 22), the plasma can be significantly larger in dimensions than the EUV beam itself, and also charging of floating surfaces to negative potential can occur up to significant distances within the vessel.

The plasma sheath is the potential drop region near the wall surface and will depend on the local plasma density and electron temperature. ${ }^{23}$ Even though care should be taken with using standard equations for sheath thickness and potential drop over the sheath since the underlying thermal equilibrium assumptions are not always satisfied, they give a good approximation for the steady-state background plasma, which is likely to dominate the average behavior over time. For a $250-\mathrm{W}$ source, the sheath thickness can be estimated to be in order of $\sim 0.1$ to $1 \mathrm{~mm}$, increasing to several millimeters away from the EUV beam; the electric field at the surface can be estimated to be up to $\sim 10 \mathrm{kV} / \mathrm{m}$ close to the beam, decreasing sharply away from the beam.

For particle contamination control, the zone around the reticle is of specific interest, as particles on the reticle have the most severe impact. ${ }^{24}$ The reticle is clamped to a scanning stage and faces downward, with metal reticle masking blades and other conductive surfaces in close proximity. The resulting slits suppress diffusion of the plasma, and transport of plasma through these slits is largely driven by the fast photoelectrons, as shown in Fig. 4. It should be noted that the EUV-induced plasma will be different for different locations in the scanner since every successive mirror in the optical system will absorb $\sim 30 \%$ of light. ${ }^{25}$ Still, as the reticle is located roughly in the center of the scanner system, this can be taken as a reasonable first approximation for the entire scanner.

At first glance these plasma parameters seem indicative of a glow discharge, but the pulsed photo-ionization origin of the plasma leads to important differences, such as strong transients and non-Maxwellian energy distribution function during and after the EUV pulse (of $<100 \mathrm{~ns}$ ). This precludes the use of fluid-like models, which rely on continuity equations for moments of the distribution functions for electron density, velocities, and energies. Instead, a kinetic model must be used that can solve the full equations for the electron distribution functions without any 
a priori assumptions about their shapes, such as (Monte-Carlo) particle-in-cell (PIC). ${ }^{26}$ The essence of this model consists of a Poisson equation solver, followed by updating the charged particles positions and velocities based on the obtained electric field distribution and individual particle velocities. This model has been tailored for simulation of EUV-induced plasma ${ }^{27}$ and validated at a relevant pressure of $5 \mathrm{~Pa}$ in an off-line test setup using an electrostatic quadrupole plasma analyzer. ${ }^{28}$ Recently, we have further extended this model to a full 3D PIC model, with options to speed up calculations by hybridization of the model with fluid-like model for the cooled electrons.

\section{Plasma and Electronics: Plasma-Assisted Discharges}

Classically, the risk of discharges is described by the Paschen criterion. This describes the condition where the gain factor by cathode electron generation plus gas ionizations by accelerating electrons exceeds the loss factor of electrons to the anode surface to trigger a self-amplifying discharge. ${ }^{29}$ This requires that electrons can gain enough energy between collisions, requiring a sufficiently long mean free path and high enough electric field but also have sufficient collisions. These considerations yield a voltage threshold as function of gas type, pressure, and distance, above which a self-amplifying avalanche effect will occur, driving the current through the gas sharply up. This can be plotted as breakdown voltage versus pressure times distance (p.d), which is called the Paschen curve (see Fig. 5). Compared to air, hydrogen has a relatively low minimum breakdown voltage of $273 \mathrm{~V}$, at $\mathrm{p} . \mathrm{d}=1.5 \mathrm{~Pa} . \mathrm{m} ;{ }^{32}$ for pressures of $\sim 5 \mathrm{~Pa}$ this translates to critical distances of $\sim 30 \mathrm{~cm}$.

For a near-vacuum system, left of the minimum, the Paschen criterion in principle allows very high voltages but care should be taken for long discharge path lines (e.g., to vessel walls), and for points of field amplification, such as a sharp edge or protrusion, or a particle, especially at the anode. ${ }^{33}$ A notable concern is coating edges, such as on both backside and frontside of the reticle-coating edge, which will have an effective submicron edge radius, resulting in significant field amplification (which can acerbated by the triple point junction of dielectric glass substrate, conductive coating, and vacuum). Also, care should be taken that AC or switching voltages can reduce the Paschen threshold. ${ }^{34}$ Given the high energy densities, a Paschen-discharge may easily both generate and release particles, mainly by local overheating at the point of contact of the electrons.

While the Paschen criterion has been proven to work well in ambient conditions (to the right of the minimum), care should be taken in (near-)vacuum, for several reasons: surface properties and feedback mechanisms become more important w.r.t. gas properties, adsorbed gases can become dominant over background gas (especially $\mathrm{H}_{2} \mathrm{O}$, but also, e.g., $\mathrm{O}_{2}$ and $\mathrm{N}_{2}$ ), and curved electrical field lines at electrode edges can lead to longer discharge paths. ${ }^{35}$ At the same time, surfaces can act both as electron sinks and (secondary) electron sources; thus, as shown in Fig. 5, the minimum breakdown voltage may be increased in presence of surfaces, but the steep slope for the low-p.d regime may also be significantly reduced: in this case, even at low p.d values,

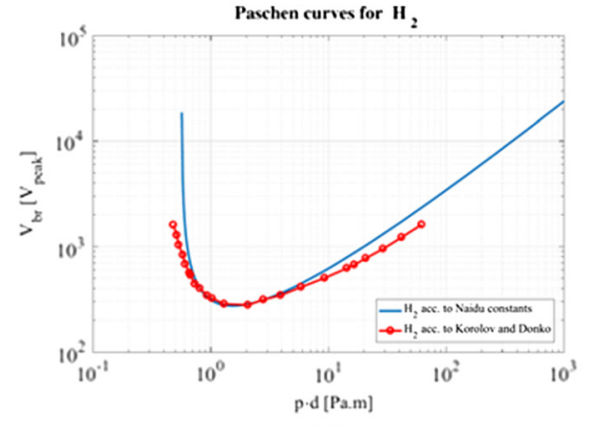

(a)

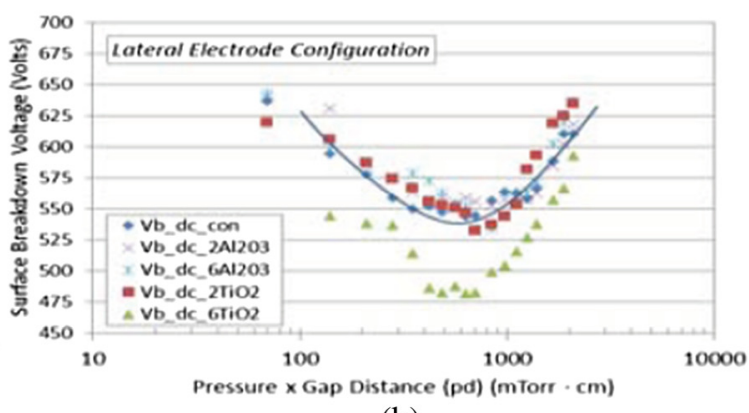

(b)

Fig. 5 (a) Paschen curve for $\mathrm{H}_{2}$, showing good match between analytical expression and measurements. ${ }^{30}$ (b) Modified breakdown curves in presence of a surface (insulating epoxy, with different nanoparticle additions), showing a gentler slope at low p.d-values (reproduced from Ref. 31). 


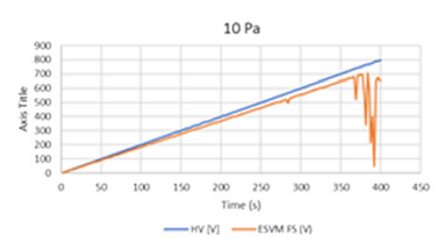

(a)

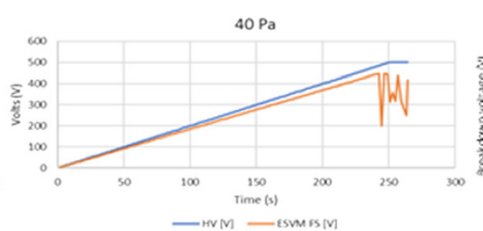

(b)

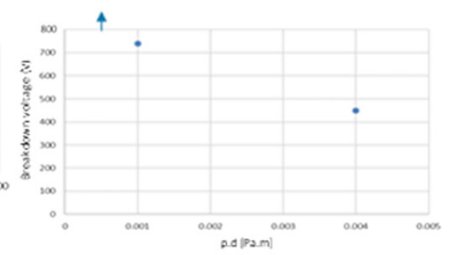

(c)

Fig. 6 (a) and (b) Gradual voltage ramp at $2 \mathrm{~V} / \mathrm{s}$ until breakdown for 10 and $40 \mathrm{~Pa}$; blue line is applied voltage to frontside, red line is frontside voltage as measured by ESVM. (c) Summary of the breakdown voltages for different p.d values (no discharge was observed at $5 \mathrm{~Pa}$ for up to $800 \mathrm{~V}$ maximum). ${ }^{36}$

critical discharge voltages may remain limited to well below $1 \mathrm{kV}$, in contradiction of Paschen prediction.

In-house experiments confirmed these trends. A slowly increasing voltage $(2 \mathrm{~V} / \mathrm{s})$ was applied to a reticle placed on top of a standard baseplate in a low-pressure $\mathrm{N}_{2}$ environment, with insulating polyimide spacers of $\sim 100 \mu \mathrm{m}$. This confirmed discharges at voltages well below the Paschen prediction and with a weaker pressure dependence than predicted by classical Paschen theory (Fig. 6), consistent with the findings in Fig. 5. Figure 6 also shows that while lower pressure allows for somewhat higher voltage, the amplitude of the discharge is larger. Tests at $5 \mathrm{~Pa}$ did not show discharges up to $800 \mathrm{~V}$.

The discharges caused particle removal from the reticle. In the experiment, the baseplate was seeded with $5 \mu \mathrm{m} \mathrm{SiO}_{2}$ particles. These were observed to be removed from the baseplate and transferred to the reticle. This is not simple electrostatic release, as one might expected for field strengths in order of $\sim 5 \mathrm{MV} / \mathrm{m},{ }^{37}$ because it is not observed for $5 \mathrm{~Pa}$ and lower pressures, which were exposed to the highest fields: up to $8 \mathrm{MV} / \mathrm{m}$. It is correlated to the local discharges that happen at pressures of 10 and $40 \mathrm{~Pa}$ at lower field strengths ( 7 and $4.5 \mathrm{MV} / \mathrm{m}$, respectively). Our proposed explanation is that the $\sim 5 \mu \mathrm{m}$ particles act as field amplification points to trigger discharges that also release the particle, but this hypothesis could not be positively confirmed due to insufficient accuracy in measuring pre- and postconditions.

When surface aspects dominate, this also implies a higher likelihood of surface flashovers versus through-gas discharges to opposite surfaces. ${ }^{38}$ For reticle discharges as outlined above, this is a concern for frontside defectivity since a flashover from the charged backside via the floating frontside to the (grounded) baseplate would increase the risk of particles being generated that can reach the frontside. In view of this, it is advised to have dissipative reticle support studs to allow the reticle to gradually decharge to the baseplate.

In presence of plasma, the free electrons and ions invalidate the basic Paschen assumptions and result in a significant shift of the avalanche threshold to lower voltages. In itself, the plasma will not focus either on electrons or ions, so local discharge-like damage such as overheating is not to be expected from plasma. However, in combination with an external voltage, current focusing can indeed occur, and such a plasma-assisted discharge can induce surface damage and create particles. This is obviously a concern for the high-voltage electrostatic clamps used in the EUV scanner, ${ }^{39}$ so these must be perfectly shielded from EUV, including the volume extending several centimeters around the EUV beam. Less straightforward is that this is also a concern for switching power supplies and circuit boards for fast sensors, which in practice can have voltages above $100 \mathrm{~V},{ }^{40}$ so these also must be properly shielded from the EUV-induced plasma.

Interaction of a plasma with biased electrodes can lead to formation of different structures. ${ }^{41}$ When the bias voltage of positively biased electrode becomes too high, formation of the so-called "fireball" structure may occur. Formation of a "fireball" in the scanner vessel is not intended, as it is very similar to a discharge, and a large current can be focused into a small area. In the presence of slits and complicated geometries, the conditions for formation of the "fireball" and similar discharges will be different as compared to bulk plasma.

We studied this with our PIC model and experimentally validated in a set-up with a simplified geometry, as shown in Fig. 7. This geometry was modeled using a fast 2D PIC model, for 


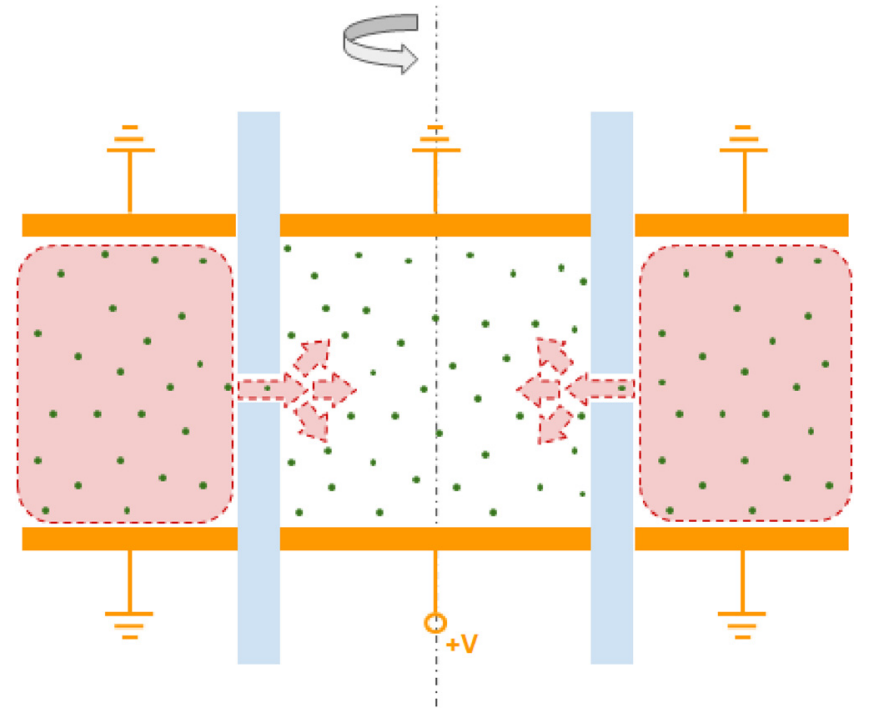

Fig. 7 Simplified 2D model geometry. Depicted are the cylindrical tube with a dielectric wall (gray), electrodes (orange), and plasma-filled region (pink). Neutral $\mathrm{H}_{2}$ gas is represented with green dots.

computational efficiency, with the same underlying physics and cross sections as our 3D-PIC model used for more realistic geometries.

The model shows a breakdown or discharge toward the positive anode when the plasma is switched on. This can be explained by electrons being accelerated toward the anode and achieving sufficient energy for further ionizations of hydrogen molecules. As the electrons are accelerated further to the positive anode, a positively charged plasma cloud is formed that screens the electrode potential and moves the zone of electron acceleration away from the electrode, thus effectively forming a channel of current, as shown in Fig. 8. This is a similar mechanism to streamer formation in tip-shaped anodes. ${ }^{42}$ Once this channel is fully formed, after $\sim 2$ to $3 \mu \mathrm{s}$, breakdown is complete as shown by the sharp increase in current.

In contrast to Paschen theory, the plasma-assisted breakdown is not determined by the product of distance and pressure: for a given distance, higher pressure and/or higher plasma power result in higher plasma electron density and lower breakdown voltage.

As Fig. 9 shows, the model shows a peaked threshold behavior in the current at the moment of breakdown for higher pressure (10 Pa); this is the "fireball" mode. For lower pressure (1 Pa), the model shows an oscillating current, but no breakdown since the number of ions formed in this case is too low to achieve sufficient screening of the positive electrode to move the acceleration zone away from the anode and form the conductive channel as above. In that case, the plasma formed will remain in an oscillatory anode glow mode.

The experimental set-up is essentially a cylindrical tube with two electrodes and a possibility to add free charge carriers from an RF plasma, as shown schematically in Fig. 10. Hydrogen pressure was varied in the range of 1 to $10 \mathrm{~Pa}$, and RF power varied between 10 and $60 \mathrm{~W}$ and the distance between electrodes was varied in range of 1 to $10 \mathrm{~cm}$. After applying a given combination of these parameters, the bias voltage on the positive electrode is scanned from 0 to $250 \mathrm{~V}$, remaining always below the Paschen minimum of hydrogen while the electrode currents were measured continuously by a Keithley 2010 multimeter.

The resulting breakdown threshold was observed to be in order of $\sim 100 \mathrm{~V}$, significantly below the predicted Paschen threshold for this configuration and even well below the theoretical Paschen minimum of $273 \mathrm{~V}$. It was also confirmed that the avalanche is directed toward the anode, and the ion current to the cathode is $\sim 10 \times$ lower. As predicted by the model, the moment of breakdown shows a sharp peak in current, after which a steady high current flows and voltage drops somewhat, as shown in Fig. 11. Varying conditions of electrode distance and pressure resulted in observations of breakdown below and around the Paschen minimum voltage, even 


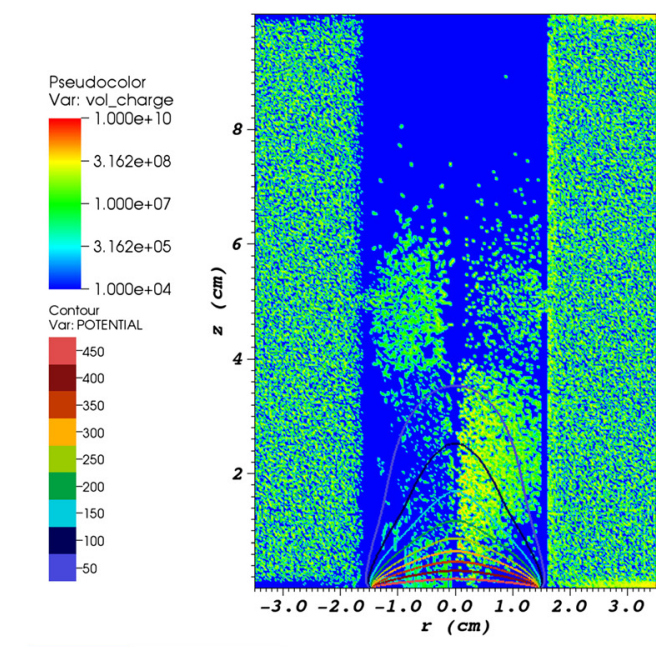

(a)

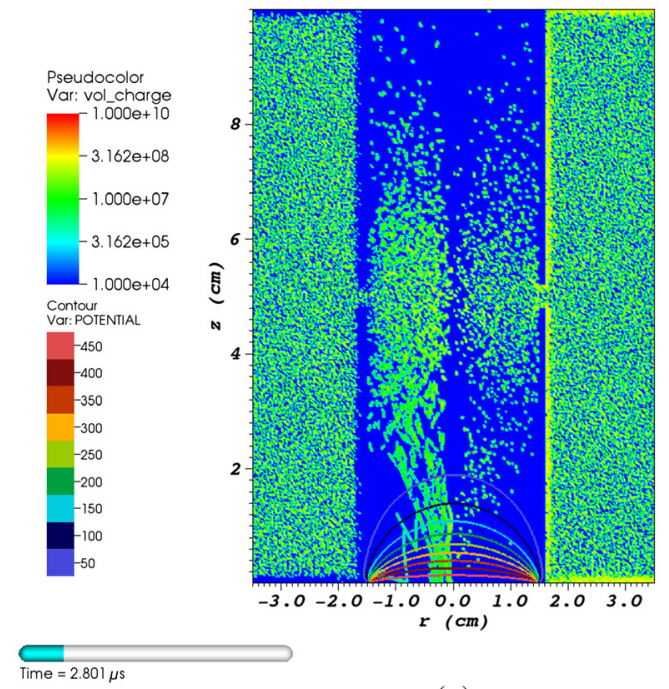

(c)

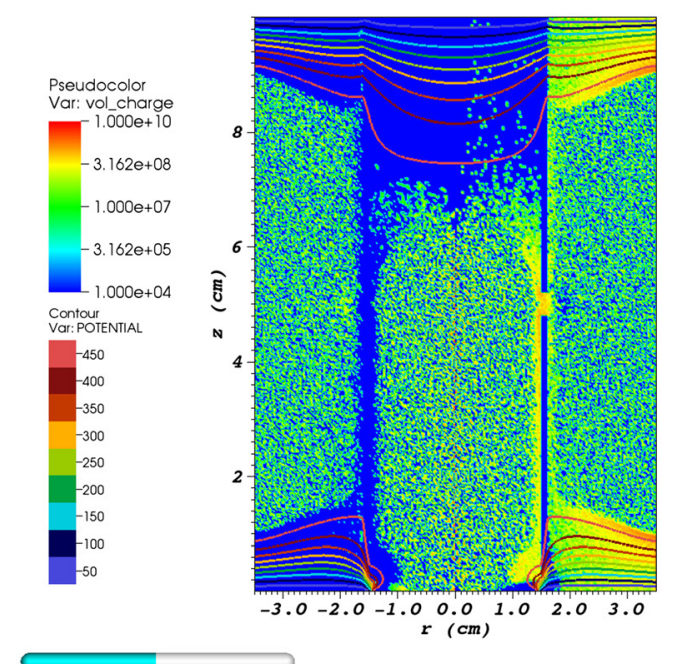

(b)

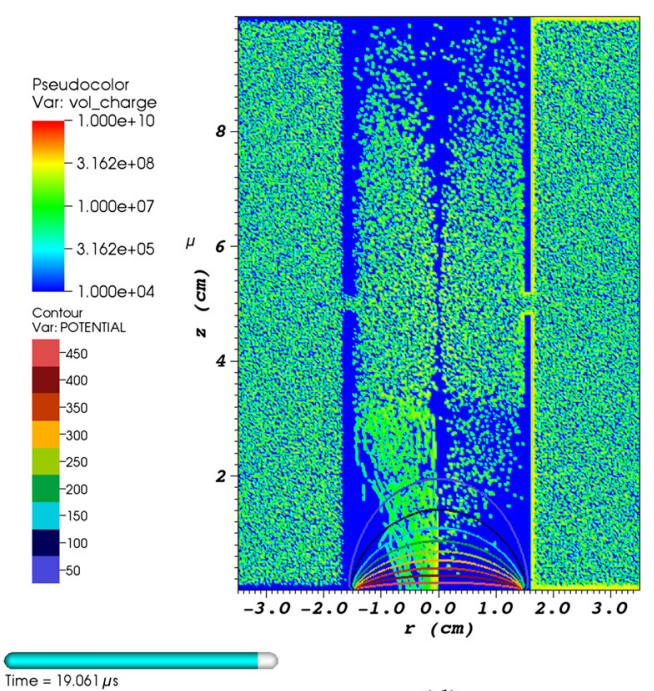

(d)

Fig. 8 Simulated plasma dynamics for (a), (b)10 Pa and (c), (d) $1 \mathrm{~Pa}$, showing electron and ion densities (left and right sides of each image) and voltage contour lines, for specific time stamps. Anode potential was set to $500 \mathrm{~V}$. The snapshots for $10 \mathrm{~Pa}$ show the transition to breakdown at $\sim 3 \mu \mathrm{s}$, whereas the snapshots for $1 \mathrm{~Pa}$ show the more or less stable anode glow.

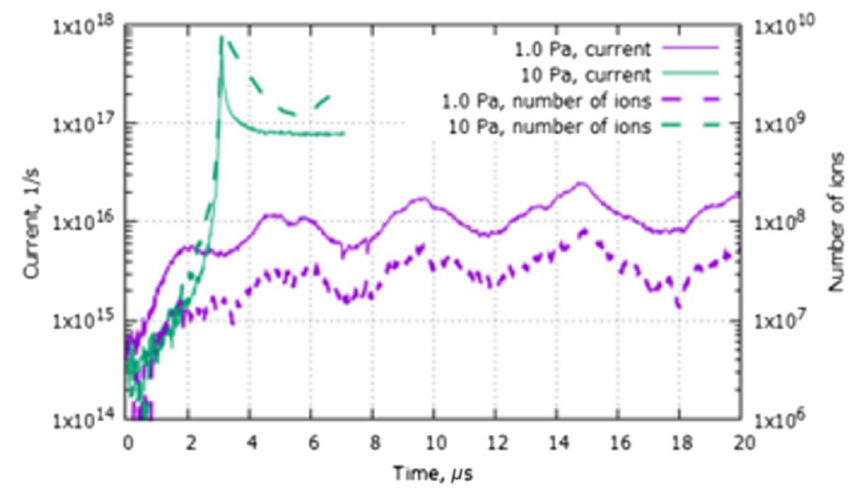

Fig. 9 Model showing avalanche and breakdown at $10 \mathrm{~Pa}$, with sharply peaked increase in current (green line) and oscillating glow discharge at $1 \mathrm{~Pa}$ (purple line). 


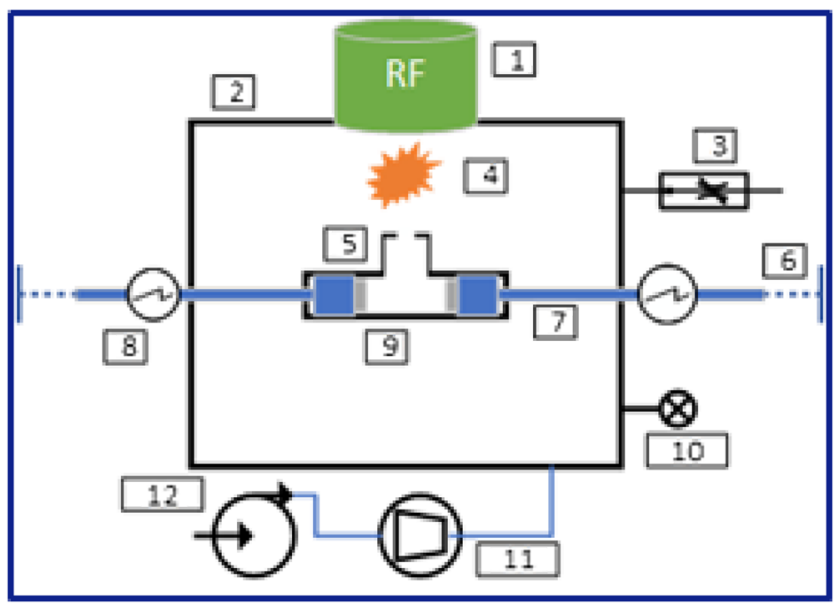

Fig. 10 Experimental setup for plasma-assisted discharge: 1, Cesar 1312 RF generator; 2, stainless steel chamber; 3 , hydrogen valve; 4 , hydrogen plasma; 5 , glass tube; 6 , micrometer translation stage; 7, insulated PTFE rod with a connector; 8, power supply/multimeter; 9, stainless steel electrode; 10, pressure gauge; 11, turbo-pump; 12, rotary pump.
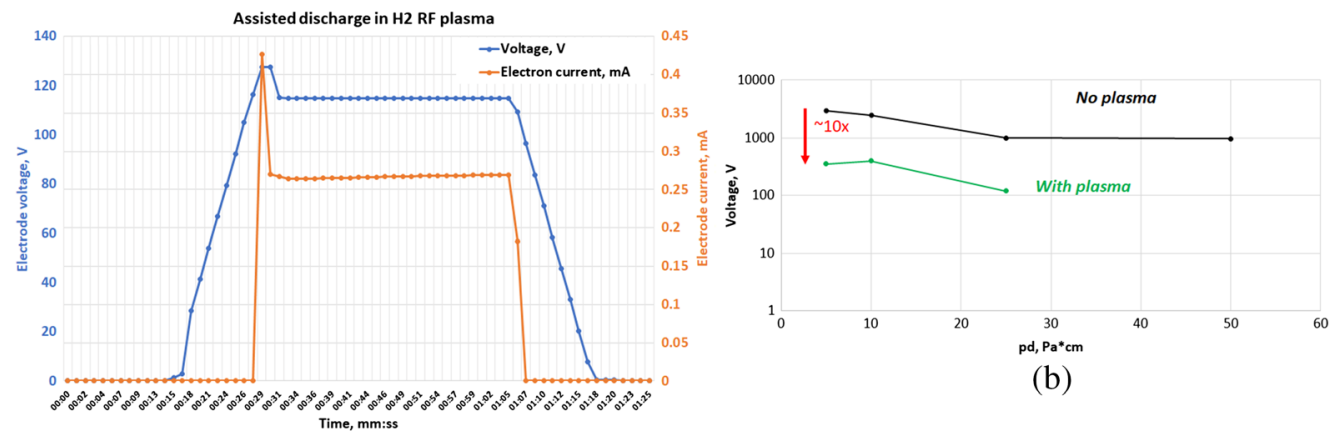

(a)

Fig. 11 (a) Example of plasma-assisted discharge and current when ramping up voltage between electrodes while plasma switched on in neighboring chamber (using $60 \mathrm{~W}$ plasma, $5 \mathrm{~Pa}, 10 \mathrm{~cm}$ distance). (b) Examples of significant reduction of breakdown voltage when plasma is switched on (10 W plasma; $5 \mathrm{~cm}$ distance; 1, 2, and $5 \mathrm{~Pa}$ ); both with and without plasma, the observed breakdown voltages at low pressures are all significantly reduced with respect to classical Paschen prediction of $>10^{5} \mathrm{~V}$ (see Fig. 6), which is attributed to the glass tube wall surfaces.

for low pressure and short distances, in what classically should be a "safe" zone of p.d-V combinations.

In general, the critical ion density $n_{i}^{\text {crit }}$ to trigger a plasma-assisted discharge can be estimated from the condition of positive electrode screening: the potential drop due to volume charge should be comparable with the electrode potential drop:

$$
n_{i}(t)>n_{i}^{\text {crit }} \sim \frac{2 \varepsilon_{0}}{e} \cdot \frac{\varphi}{h^{2}} \sim 10^{8} \mathrm{~cm}^{-3} .
$$

With the electrode potential drop $\varphi \approx 30$ to $70 \mathrm{eV}$ (to accelerate electrons to energies at which ionization is most efficient ${ }^{43}$ ), the region of ion accumulation $h \approx 1 \mathrm{~cm}$, and $e$ is the elementary charge. This estimate of $n_{i}^{\text {crit }}$ is in line with the 2D PIC simulations above. In more complicated geometries, the exact value of the critical ion density will depend on the (in)homogeneity of the electric field and the distance of the plasma source to the anode. Still, rather than a discharge threshold being determined by voltage and the product of pressure and distance, as in the case of Paschen, the discharge threshold is now driven by applied voltage $V_{\text {ext }}$, pressure $p_{\mathrm{H} 2}$, and local plasma density, which in turn scales with pressure and EUV power $I_{\mathrm{EUV}}$ and with a suppression factor $\gamma_{\text {supp }}$ that describes the fall-off of plasma away from the EUV-beam: 


$$
n_{i}^{\text {crit }} \sim V_{\mathrm{ext}} \cdot p_{\mathrm{H} 2}^{2} \cdot I_{\mathrm{EUV}} \cdot \gamma_{\mathrm{supp}}
$$

Equation (2) illustrates that the risk of plasma-assisted discharge needs to be re-evaluated for any increase in either EUV power, local pressures, or external voltages. For the complicated internal geometries of an EUV-scanner, no analytical expression can be used. However, our 3D PIC model, with the same underlying physics and cross sections as the 2D PIC model as used and validated above, can now be used to check any design proposal for safe limits on local voltages and pressures plasma-assisted discharges.

Also, as general guidelines, floating or insulating surfaces should be avoided as much as possible to minimize the risk of surface flashovers, and edges and protrusions should be sufficiently rounded to avoid dangerous field amplification points.

\section{Reticle Charging and Discharges}

As outlined in Sec. 3, floating surfaces and dielectrics close to the EUV-induced plasma may become charged. In particular, the reticle needs to be considered in this respect since it consists of an insulating glass substrate with a conductive coating on the backside (for electrostatic clamping purposes), and a conductive reflective multilayer coating on the frontside; both conductive layers are floating independently; this is outlined in Fig. 12.

The reticle backside is clamped electrostatically to a movable positioning module, whereas the frontside is directly exposed to the EUV beam and EUV-induced plasma. Grounding of the reticle is impractical in view of the risk of particles generated when making electrical connection through the oxide top layers on the moving/scanning reticle. ${ }^{45}$ During exposures the reticle frontside will acquire a transient potential due to competing direct photoelectric effect from EUV irradiation (driving to positive) and subsequently decharging from plasma and will return to $\sim 0 \mathrm{~V}$ after every pulse, as shown in Fig. 13 .

The reticle backside is quite different since this is not exposed directly to EUV irradiation and shielded by the clamp. The backside coating plane is connected to the plasma volume through only a small gap, which acts as a spatial filter to suppress diffusion for both positive and negative charges. Still, two effects can result in charging of the reticle backside: and secondary gas ionizations and secondary electron emission by the surrounding clamp, of which secondary gas ionizations are expected to be dominant.

Secondary ionizations in the gas surrounding the reticle by the energetic photoelectrons result in electrons propagating more or less isotropically around the actual EUV beam, which allows electrons to reach the conductive backside coating even if the coating is recessed from the edge. The ions have a lower likelihood to reach the backside as these are accelerated more along the electrical field lines, scatter less, and have higher inertia; so ions will likely hit surrounding surfaces and stick there. This results in a net negative charging of the reticle backside, as is shown schematically in Fig. 14.

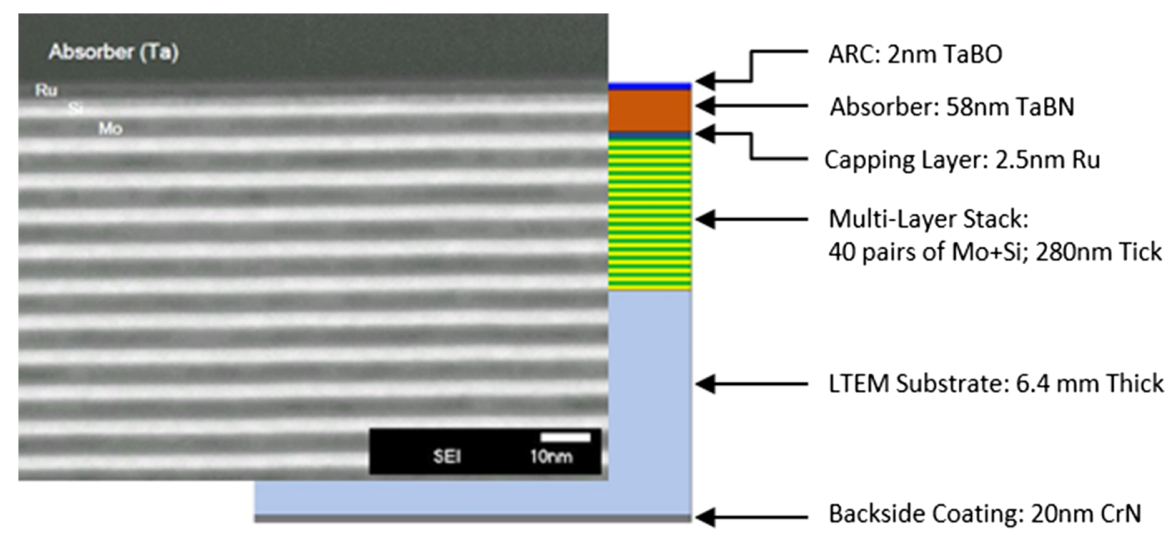

Fig. 12 Sketch of reticle cross-section, showing conductive coating stacks on frontside and backside; adapted from Ref. 44. 


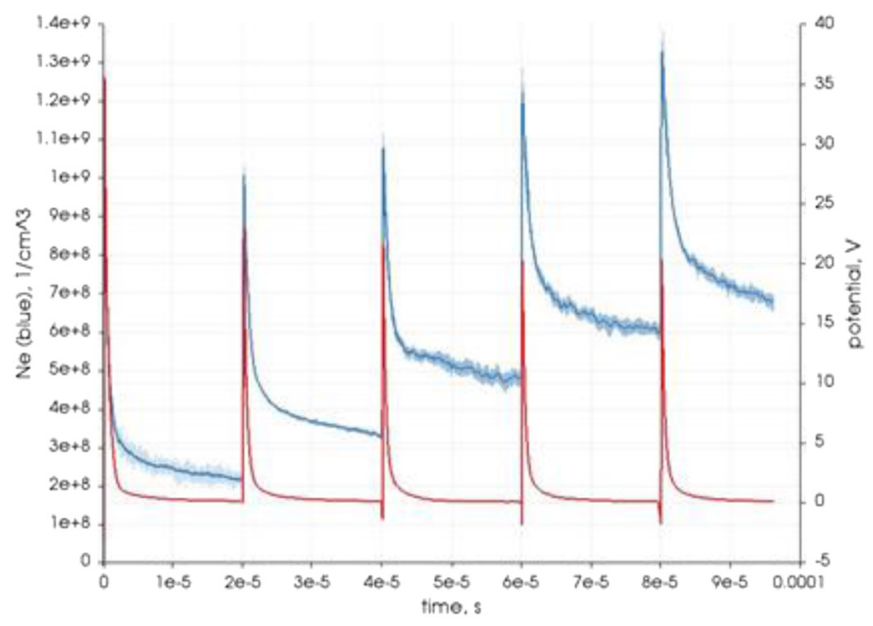

Fig. 13 PIC simulation of electron density (blue line) and reticle frontside surface potential (red line), showing accumulation of plasma over pulses but no build-up of surface potential.

(a)

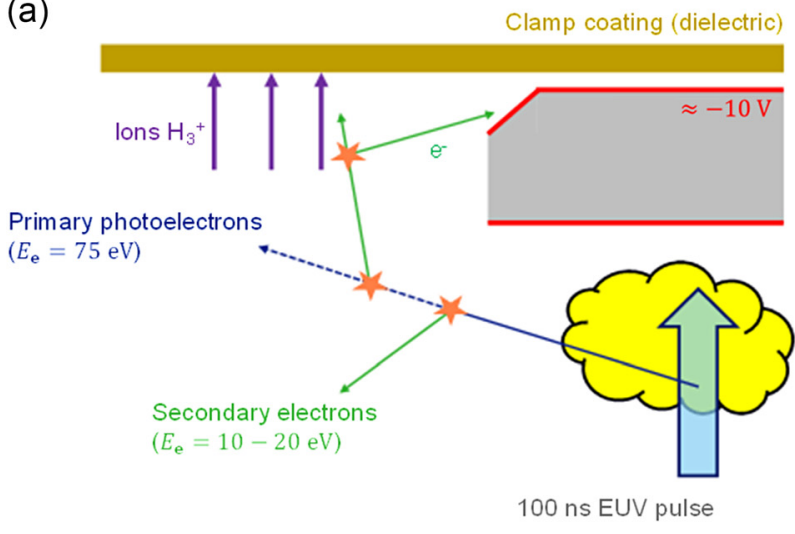

(volume) collisions

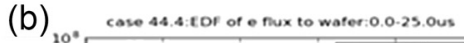

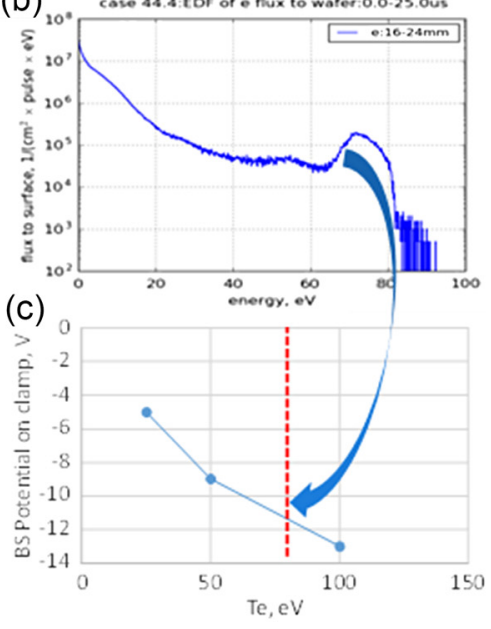

Fig. 14 (a) Basics of charging mechanism of reticle backside; (b) energy spectrum of electrons reaching reticle edge modelled by $3 \mathrm{D}$ PIC code. (c) Energetic secondary electrons charge reticle backside while ions are carried by field and momentum toward nearest wall resulting in a net negative charge on reticle.

As the reticle backside charge and voltage build up, electrons will be repulsed and ions attracted, which will result in an equilibrium charge and voltage, which will depend on details of plasma (e.g., EUV power, pressure, and beam position with respect to reticle edge). PIC modeling for the reticle geometry in NXE:3400 of electron spectrum reaching the backside reticle edge shows that the cumulative process of charging by fast electrons and partial neutralization by ions result in an equilibrium negative voltage in the order of $-10 \mathrm{~V}$. This voltage, and the associated excess electrons, will remain on the reticle backside after the plasma fully decays at the end of exposures. The backside voltage has been simulated to scale inversely quadratically with increasing pressure, as shown in Fig. 15; this is due to the combined effect of reduced average frontside potential and increased collisions at higher pressure, which act to reduce the highenergy tail of the EEDF.

Also, reticle geometry and coating details are relevant: a recessed coating will show a higher equilibrium charge since this will increase the spatial filtering of ions and more so than for electrons (due to secondary electron emission or bouncing of electrons from surfaces). This implies an additional consideration for the reticle backside coating beyond the existing specifications for clamping, with the coating preferably extended as close as possible to the edge. The acquired 


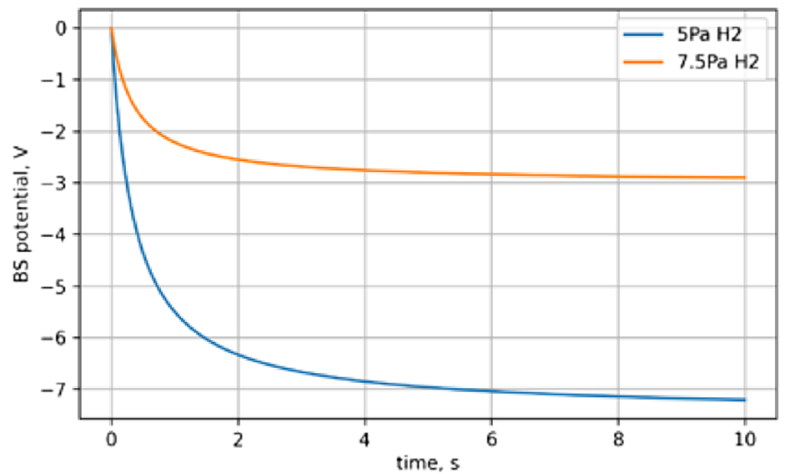

Fig. 15 PIC simulations of backside voltage dependence on time and pressure, showing $>2 \times$ reduction in voltage for $1.5 \times$ higher pressure.

backside voltage may seem negligible, but during reticle unloading the backside voltage is amplified by the changing capacitance between reticle and clamp, as outlined in Fig. 17, whereas the charge locked onto the floating surface remains constant. ${ }^{47}$ This is reflected in the basic equations:

$$
\begin{aligned}
& U=\frac{Q}{C}=\frac{Q \cdot d}{\varepsilon_{0} \cdot A_{\mathrm{cl}}}, \\
& U_{\mathrm{unl}}=U_{\mathrm{cl}} \cdot \frac{d_{\mathrm{unl}}}{d_{\mathrm{cl}}},
\end{aligned}
$$

where $A_{\mathrm{cl}}$ is the (constant) area of the clamping electrode, $U_{\mathrm{unl}}$ is the backside potential during unload, $U_{c l}$ is the backside potential as clamped, $d_{\mathrm{cl}}$ is the distance as clamped, which is in order of microns, and $d_{\text {unl }}$ is the distance during unloading, which is in order of centimeters (as sketched in Fig. 16), giving a potentially $\sim 1000 \times$ increase.

In reality, capacitive coupling between backside and frontside and to the unloading plate will complicate this relation and limit the voltage amplification to about $50 \times$, as shown in Fig. 17; this still means that during unloading the backside potential can reach a value of up to $1000 \mathrm{~V}$. This is well above the Paschen minimum of $\sim 275 \mathrm{~V}$ for $\mathrm{H}_{2}$, implying a risk of electrostatic breakdown and discharges.

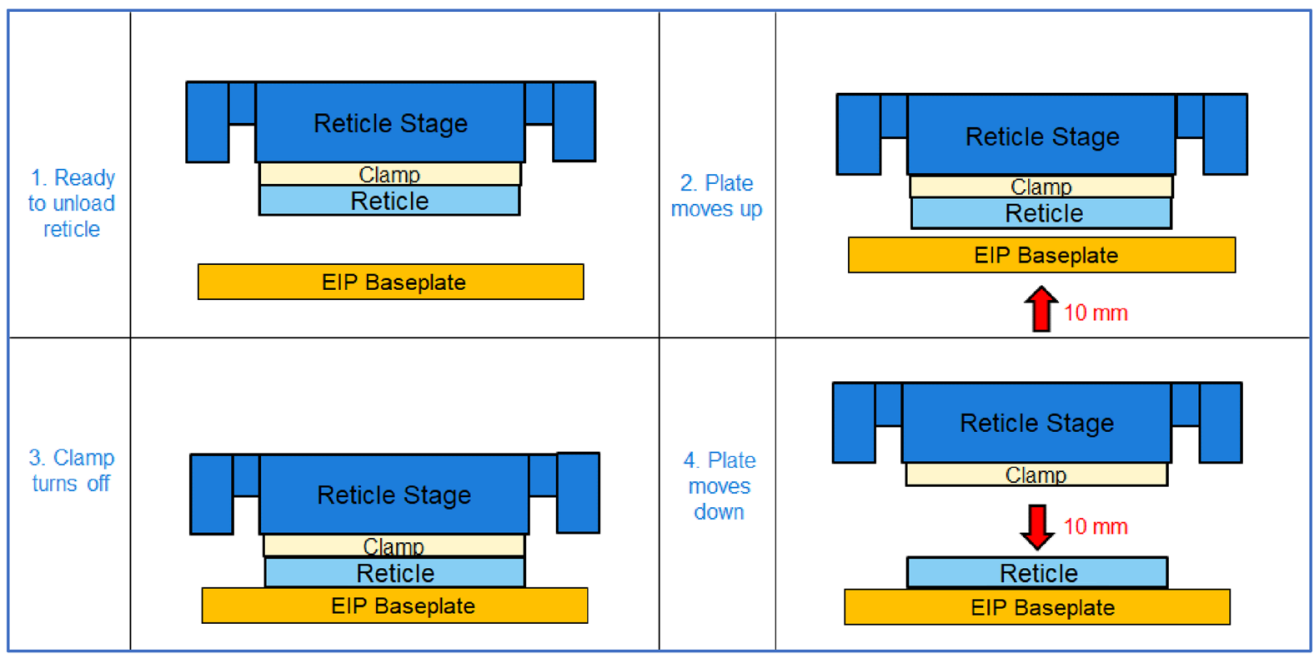

Fig. 16 Illustration of reticle unloading sequence. While clamped the separation between reticle backside and clamp electrode is in order of a few micrometers, resulting in strong capacitive coupling; during unload the separation is increased to $\sim 1 \mathrm{~cm}$, reducing the capacitance by several orders of magnitude. 


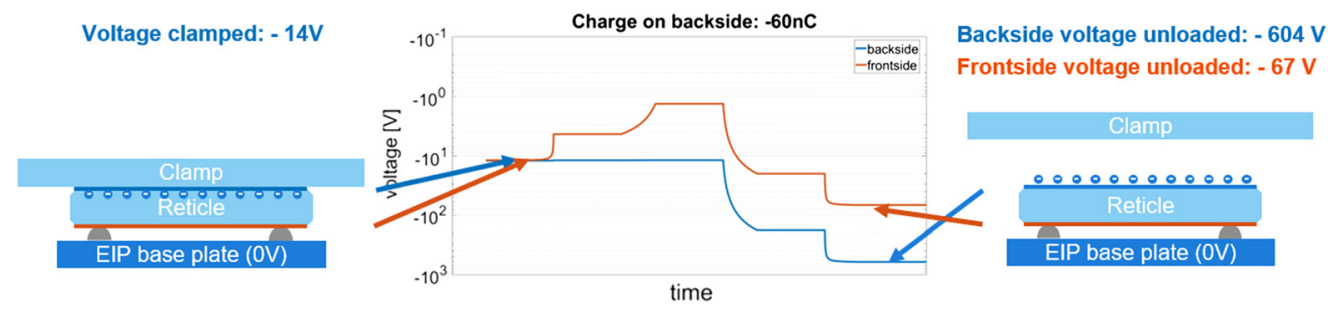

Fig. 17 Example of backside (BS) potential amplification from $\sim 14$ to $\sim 600 \mathrm{~V}$ during reticle unload (blue line), caused by stepwise increasing gap between reticle and clamp; also shown is the induced frontside voltage (red line). ${ }^{46}$

The high level of reticle charging has been confirmed by electrostatic voltage measurements (ESVM), using dual Trek PD15035 555P-style probes with a 6000B-6 sensor (in combination with a modified reticle pod to allow simultaneous access of the probes to reticle front and backside), directly after unloading the reticle from the scanner. Figure 18 shows comparison of reticle backside voltage measured after full reticle cycle through EUV machine with and without EUV exposures. Test reticles exposed to EUV confirm the high voltage of $\sim 600 \mathrm{~V}$, whereas reticles that were not exposed to EUV remained neutral. Optical microscope inspection of these test reticles indicated cosmetic damage of reticle backside coating after EUV exposures, which could be traced back to imperfections in the coating edges on the test reticles used but also showed a clear sensitivity to backside discharges from these high backside voltages.

Besides this backside discharge risk (which might in practice be acceptable since the backside is not as critical as the imaging frontside of the reticle), the increase of backside voltage during unload also induces a frontside voltage in order of $70 \mathrm{~V}$ by their capacitive coupling (red line in Fig. 17), which might not be so high as to cause concerns for discharges to the critical reticle frontside surface but is a concern for particle attraction to the reticle, as demonstrated by Amemiya (see Fig. 19). ${ }^{49}$

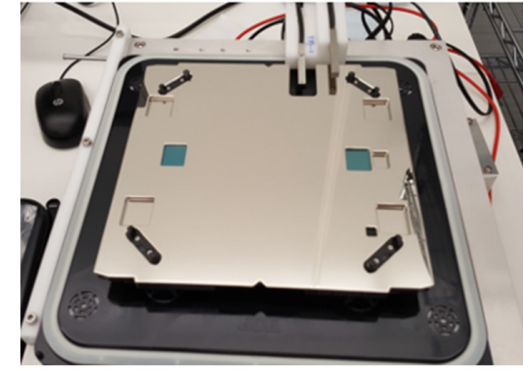

(a)

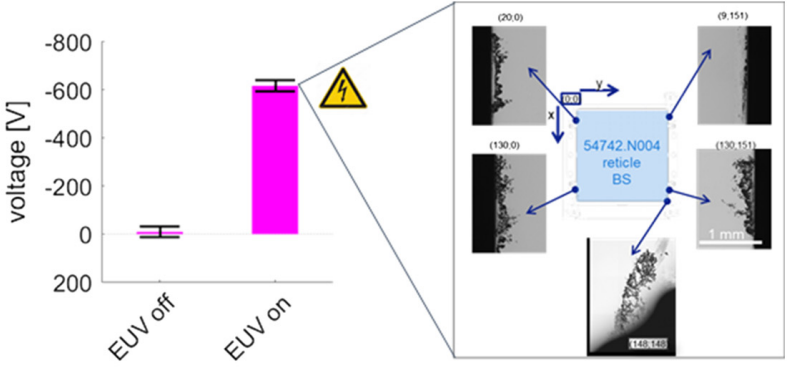

(b) (c)

Fig. 18 (a) Modified reticle pod for ESVM measurements; (b) ESVM measurement of high voltage on reticle backside when reticle has been exposed to EUV; (c) observation of ESD damage on a test reticle.

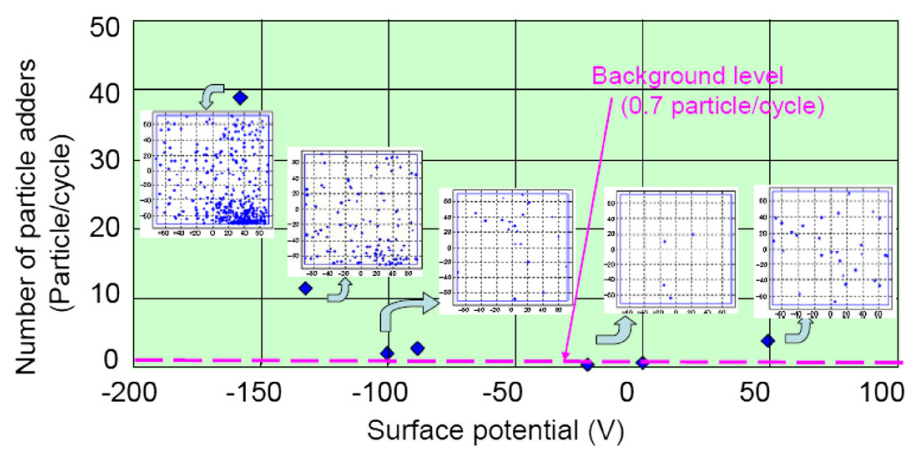

Fig. 19 Particle pick-up as a function of reticle surface potential; from Ref. 48. 


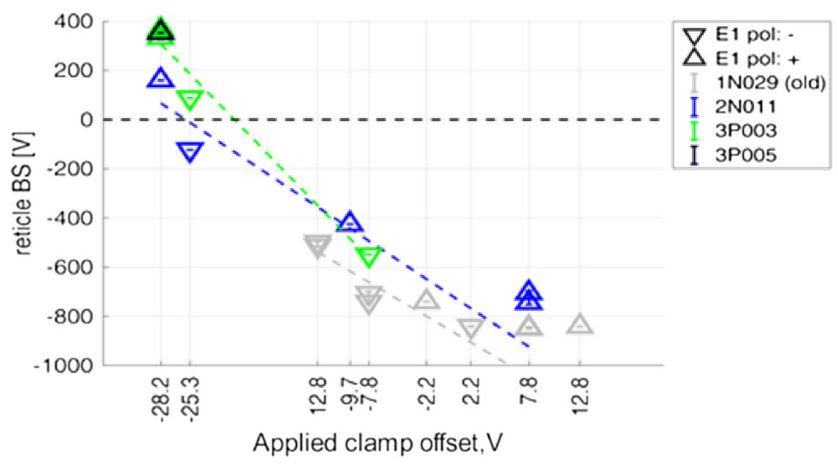

Fig. 20 Reticle backside voltage as function of clamping offset; the colors denote different specimens of test reticles from two different suppliers.

Even while the EUV reticle pod is designed with electrostatics in mind (e.g., metal inner body to prevent ESD risk as present in DUV pod ${ }^{48}$ ), the pod does not fully resolve this: frontside grounding has to be soft to prevent particle generation from hard grounding contact, so it will make poor electrical contact through the insulating top oxide of the reticle frontside coating for low voltages. ${ }^{50}$ Although the backside pod cover itself is grounded, this does not make grounding contact to the reticle backside within the scanner vacuum system or the internal reticle library but only makes contact when the EUV pod is locked at the load port to be removed from the scanner. It should be considered that this grounding is by soft contact to a potentially oxidized backside coating, so this contact might be poor in practice and should not be relied upon for backside decharging.

The issue of backside voltage excursions during unload can be remedied in two ways: (1) by creating a (negative) offset in the clamping scheme to shift the equilibrium of the EUV-plasma charging to (near) zero during the exposures or (2) by supplying free-charge carriers during the unload sequence to dynamically reduce the charge on the reticle as the voltage builds up.

A negative clamping offset has indeed been observed to result in lower backside voltage, with near-zero backside voltages during unload being achieved for an offset of roughly $-25 \mathrm{~V}$, as shown in Fig. 20, using ESVM. However, as can also be seen in Fig. 21, for reasons of reticle chamfer and coating tolerances, this offset would need to be calibrated per reticle to guarantee sufficiently low voltage at unload. The observed limit at $\sim-800 \mathrm{~V}$ is most likely an artifact of the ex situ ESVM measurements: ESVM can only be done outside of the scanner, after fully unloading the reticle, and voltages above $\sim 800 \mathrm{~V}$ are expected to result in discharges during the unloading of the reticle to ambient conditions.

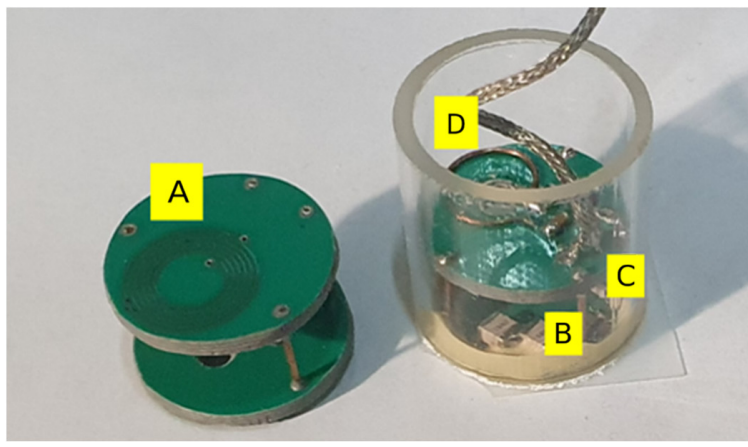

(a)

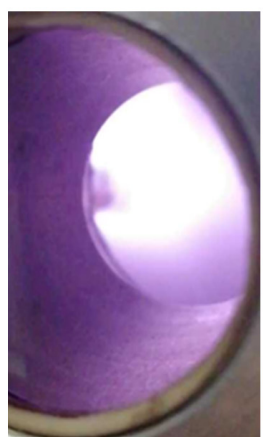

(b)

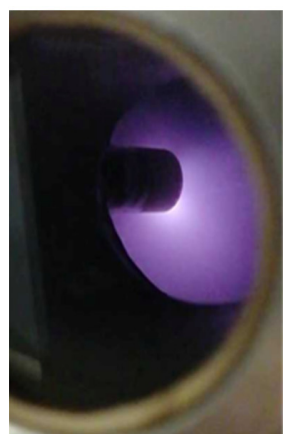

(c)

Fig. 21 (a) Prototype of miniature inductively coupled plasma generator, with (A) inductive coil, (B) high-Q capacitors, (C) impedance matching, and (D) RF current monitoring coil (only for prototype). The diameter of the assembly is $\sim 2 \mathrm{~cm}$. (b) Ignition of the discharge at $3 \mathrm{~Pa}$ hydrogen. (c) Stable operation at $2 \mathrm{~W}$ RF power and $3 \mathrm{~Pa}$ hydrogen. Images courtesy of ISAN. 
An alternative solution is dynamic charge compensation during the unload sequence by creating a supply of free-charge carriers; this will reduce the charge on the reticle as the voltage builds up and thus will maintain acceptably low voltage levels throughout the unloading sequence to prevent any risk of discharge.

This could be achieved by a miniature plasma generator, such as proposed and developed by Hopwood $;^{51}$ though such a device has been shown to work for hydrogen, ${ }^{52}$ the additional hardware is hard to retrofit into the existing scanner modules and reliably igniting the hydrogen plasma at $\sim 2 \mathrm{~Pa}$ is still considered a challenge. Figure 21 shows a prototype demonstration of such a device.

Dynamic charge compensation can also be achieved by turning on the EUV-induced plasma during the unload sequence, which has been termed "EUV@unload." 53 During the unload sequence, the reticle is moved to a position next to the EUV beam, is placed onto a baseplate, and subsequently lowered from the clamp. As the gap between reticle and clamp increases, the capacitance drops and the negative backside voltage builds up, attracting the ions from the EUVplasma; simultaneously the opening gap allows the ions to reach the backside coating more easily to reduce the net charge. Even though the reticle is moved several centimeters away from the EUV beam during unload, the ions are pulled toward the high negative potential on the reticle backside, which develops as the reticle is moved away from the clamp.

It should be noted that volume recombination is very low at this low ionization degree and pressure, so ions can travel a long distance if the directional motion in the electric field is stronger than diffusion to the walls. This is shown in Fig. 22. Full scanner tests confirmed that the EUVinduced plasma density is sufficient to counter the voltage amplification effectively, without delays or slowdowns in the unload sequence. Besides being relatively insensitive to reticle tolerances, this also has key benefits in using pre-existing hardware and having no ignition threshold. Therefore, this scheme has been chosen as baseline for the current generation of EUV-scanners.

Recent customer data have shown that EUV@unload significantly suppresses defectivity associated with electrostatic pick-up from the reticle pod baseplate, such as carbon-based fall-on particles, without deterioration of other particle types. Also, in-house testing on system (which had a known grounding issues) showed the effectiveness of EUV@unload to mitigate the resulting defectivity issue, as shown in Fig. 23.

Although current performance of the EUV@unload scheme is satisfactory, further improvements or accelerations are currently being investigated. One option could be to combine EUV@unload with clamp electrode biasing as outlined above, or alternatively to apply a negative bias voltage to the clamp during unloading to attract more ions from the EUV-plasma. For the long term, it is recommended to investigate grounding of both reticle surfaces during scanning; one option could be to ground the backside via hard electrical contact to the clamp and to create electrical connection between backside and frontside coatings.

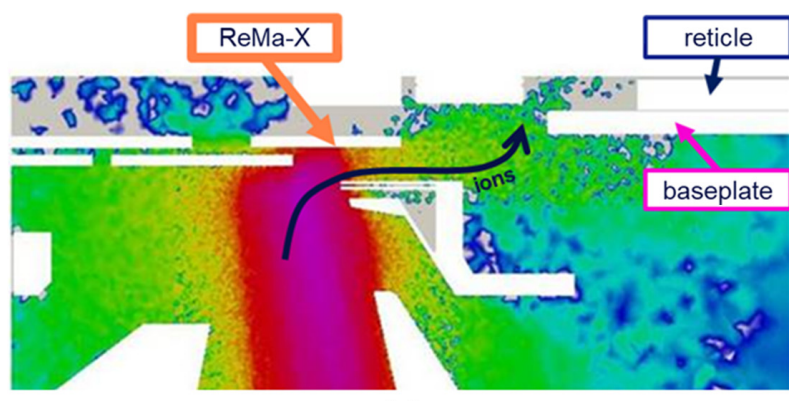

(a)

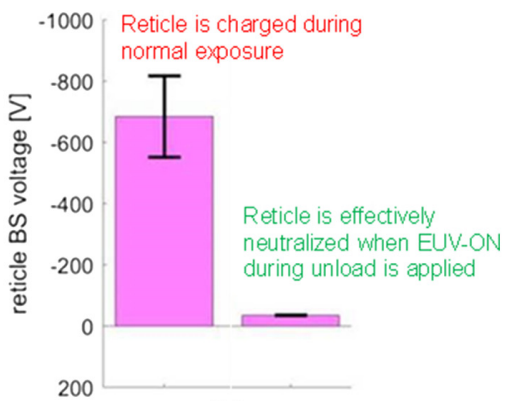

(b)

Fig. 22 EUV-plasma now neutralizes reticle backside during reticle unload, even with the EUV beam several cm's away. (a) Reticle location with respect to EUV beam and modeled ion densities; (b) reticle backside voltages measured by ESVM, without and with EUV on during unload. 
Chart Title

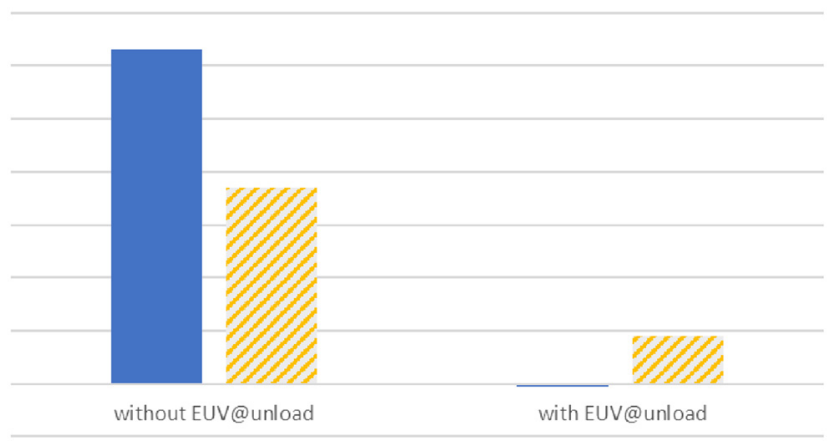

Fig. 23 Effective mitigation of ESD-related particles (solid blue) by EUV@ unload; 4× improvement in non-ESD, or fall-on, particles (orange striped) cannot be attributed to EUV @ unload but is likely due to flushing in between the two measurements.

\section{Particle Transport and Reticle Protection}

Another electrostatic aspect of EUV is that, as shown above, during the EUV pulse the floating reticle surface will instantaneously charge positively by photoelectric effect to 20 to $40 \mathrm{~V}$ and subsequently will be neutralized within $\sim 5 \mu$ s to $0 \mathrm{~V}$ by charge compensation from the EUVinduced plasma. This process repeats after $20 \mu$ s with the next pulse. So on average, the reticle will be charged $\sim 1$ to $2 \mathrm{~V}$ positively with respect to the surrounding grounded surfaces. As the reticle top layers ( $\mathrm{Ru}$ cap and $\mathrm{Ta}$ absorber) are conductive and continuous, all of the reticle will take on this average positive potential, also the (large) part of the reticle that is away from the actual EUV-beam. Away from the EUV-beam, plasma density is too low to effectively shield the resulting electric field between reticle and the nearby grounded reticle masking blades, which can be in order of $>100 \mathrm{~V} / \mathrm{m}$ given the small gap.

Free particles are preferentially charged negatively in and around the EUV-beam, ${ }^{54}$ although there might be transient phase of positive charging by photoelectric effect. ${ }^{55}$ This results in an attractive electric force between reticle and particle. Charging of free particles inside the EUV beam will show transients with the EUV pulses, first charging positively by photoelectric effect, then negatively due to the higher mobility of the plasma electrons, and subsequently (partly) neutralizing due to the ions, as described by orbital motion limited (OML) theory. ${ }^{56}$ Assuming thermal equilibrium, OML provides the steady state potential of the particle $\phi_{p}$ when the electron and ion fluxes are balanced:

$$
\exp \left(\frac{e \phi_{p}}{k_{B} T_{e}}\right)=\sqrt{\frac{m_{e} T_{i}}{m_{i} T_{e}}}\left(1-\frac{e \phi_{p}}{k_{B} T_{i}}\right)
$$

where $m_{e}, m_{i}$ and $T_{e}, T_{i}$ are the masses and temperatures of the electrons and ions, respectively. With the ions close to room temperature, the particle potential is mainly determined by the electron temperature. Approximating the particle by a sphere, the particle charge $Q_{p}$ follows from the potential via the capacitance of a sphere, and scales linearly with the particle diameter $d_{p} .{ }^{57}$

$$
Q_{p}=2 \pi \epsilon_{0} \cdot d_{p} \cdot \phi_{p}
$$

In the transient EUV-induced plasma, no analytical equations exist for the potential or charge of a free particle, and PIC modeling is used to determine the evolution of particle charge over time. PIC simulations of the EUV-induced plasma in the region below the reticle show that micron-sized particles get a short positive charge, then flip to a negative charge, after which they reach an equilibrium between electron and ion currents, as shown in Fig. 24; this process will be reset for every new pulse. For submicron particles, reaching equilibrium between electrons and ions will typically take longer than the pulse interval, and negative particle charge will build up over multiple pulses until an equilibrium is reached between the photo-ionization and 


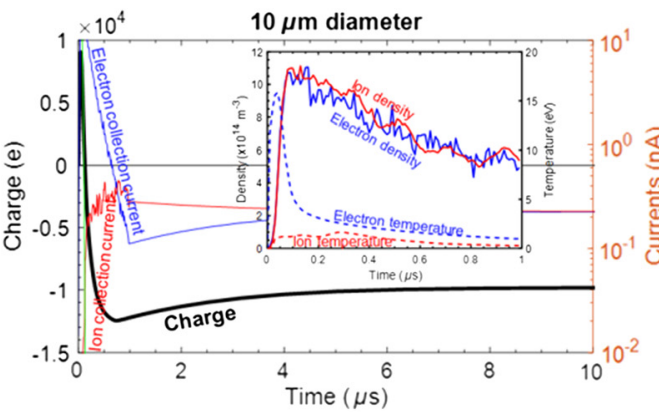

(a)

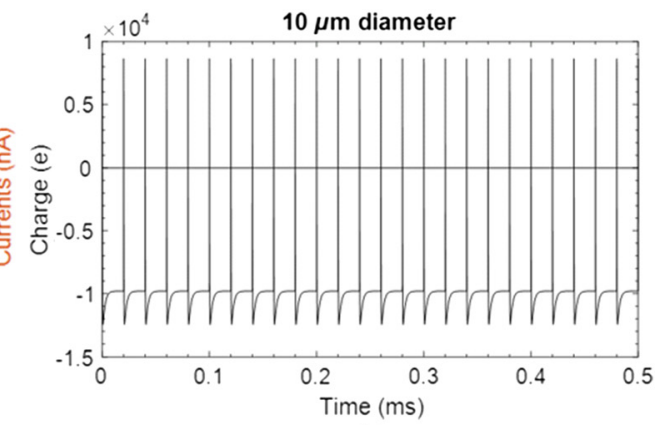

(b)

Fig. 24 (a) PIC model of charging of a $10 \mu \mathrm{m}$ particle in the EUV beam, showing fast transient positive photocharging and subsequent negative charging (to $\sim 1$ e per $\mathrm{nm}$ ); quasisteady-state charge (balanced electron and ion currents) is achieved within $\sim 5 \mu \mathrm{s}$. The inset shows the electron and ion temperatures. (b) Repeating charging pattern over multiple pulses.

the electron currents, as shown in Fig. 25. For an electron temperature of $\sim 0.5 \mathrm{eV}$, the equilibrium particle charge is predicted to be roughly $\sim d_{p}$ [e], where $d_{p}$ is the particle diameter in $\mathrm{nm}$.

Free particles next to the EUV beam will not experience the initial photoelectric effect, so they will charge negatively by the more mobile electrons and will reach an equilibrium between electron and ion collection currents; this results in a similar steady-state value negative charge as for particles within the beam or somewhat more negative for submicron particles. Further away from the beam, plasma density will drop and the charging will be much slower but will still result in an average negative charge (again due to the more mobile electrons).

Extending the PIC simulations for particle charge with dynamic reticle potential and resulting electric fields yields an electric force on the particle near the reticle surface. Comparing the resulting electric force against the other forces that might work on a free-floating particle [gravitational force, neutral, and ion drag forces, and for completeness, thermophoretic force (driven by temperature differences between the irradiated reticle surface and reticle-facing masking blades)], it is clear that the dominant forces for submicron particles are the electric force and the neutral drag force, as shown in Fig. 26.

Combining the vector force fields of electric and neutral drag forces, particle trajectories can be calculated, as shown in Fig. 27. This allows to design the local flows and pressures such that no particles larger than a given critical size $(50 \mathrm{~nm}$ in this example) will reach the reticle frontside surface. The most effective optimization parameter is pressure: increasing pressure will increase neutral drag force ${ }^{58}$ and reduce the electric attraction force (as outlined above) but will come at expense of EUV transmission. Increasing flow in itself will increase neutral drag force and does not affect the electric force, but in practice flow is not independent of pressure.

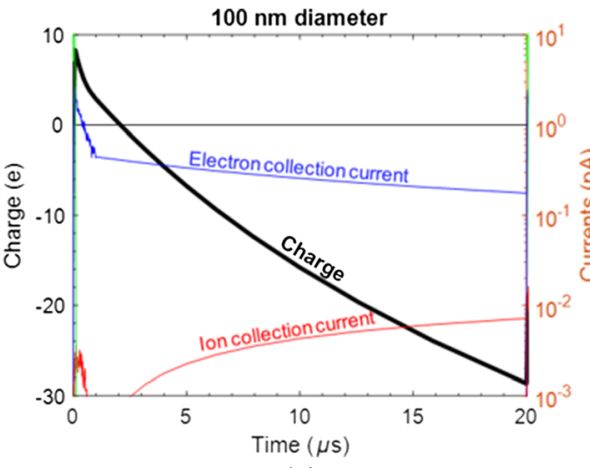

(a)

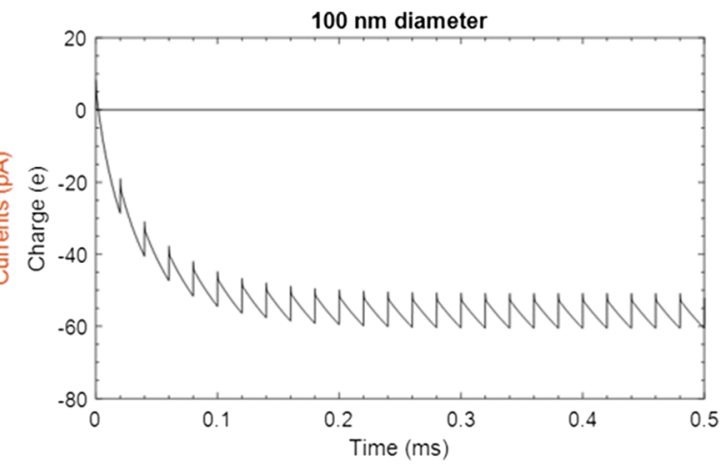

(b)

Fig. 25 (a) PIC model of charging of a 100-nm particle in the EUV beam, showing fast transient positive photocharging and subsequent negative charging. (b) Increasing charge for over multiple pulses for $100 \mathrm{~nm}$ particle. 


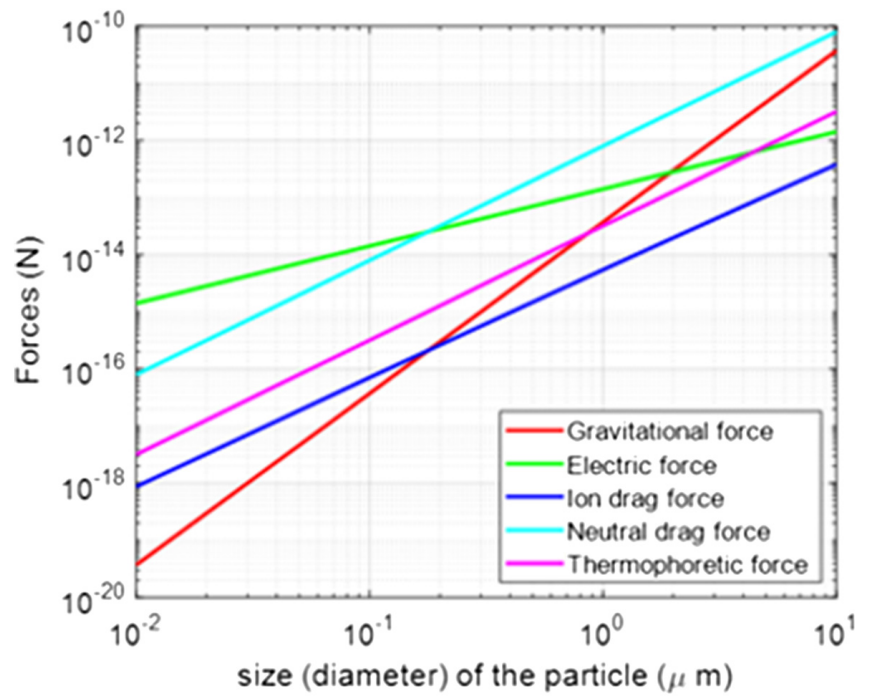

Fig. 26 Volume force estimates for submicron particles in the gap between reticle and grounded reticle masking blades, for $250 \mathrm{~W}$ source and $5 \mathrm{~Pa} \mathrm{H}_{2}$.

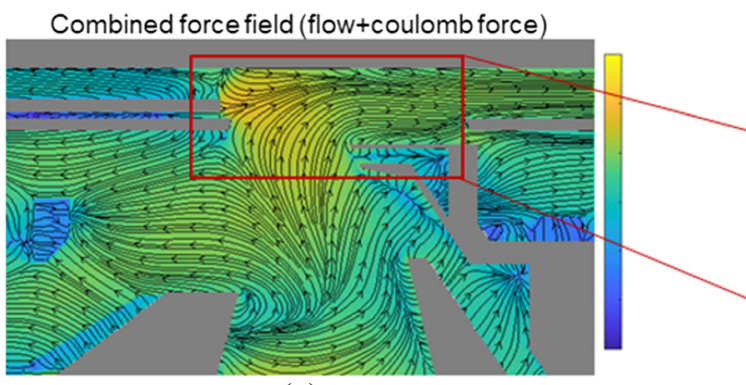

(a)

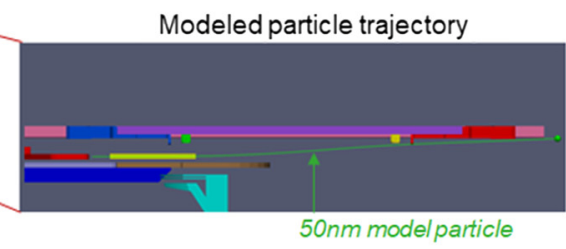

(b)

Fig. 27 (a) PIC simulations of the force map for a 50-nm diameter particle in the reticle zone, just after the EUV pulse (180 ns). More yellow means higher force (blue means lower); arrows indicate local direction of force. (b) Modeled trajectory for a 50-nm test particle.

\section{Conclusion}

Understanding of the specific nature of the EUV-induced scanner plasma and the interaction of this plasma with surfaces and particles has improved significantly in the past years. This has allowed scanner design optimizations as well as targeted improvements in manufacturing and cleaning processes, for both plasma and related electrostatics aspects, to continue to drive down the corresponding contributors behind defectivity. Analysis of plasma-assisted discharges has resulted in design guidelines for allowed voltages and pressures at various distances from the EUV-beam, to prevent discharges and improve the robustness of high-voltage electronics. Prevention of high reticle charging during unloading and handling, with the associated risk of discharges, removes a potential source of particles. These measures have brought particle contamination control of the EUV-scanner to a regime where customers have the freedom to operate without protective pellicle for high-volume manufacturing. For future EUV lithography systems, continued improvements in plasma models and understanding will ensure plasma-aware designs that will be compatible at increasing source powers and reducing critical particle sizes.

\section{Acknowledgments}

The authors wish to thank the ASML Research and Development teams for Scanner Plasma and Defectivity for scanner testing, fruitful discussions, and general support. Also, we would wish to thank Ronald van der Wilk, Alex Yanson, Frank van Lier, and Jeroen van Duivenbode for the 
electrostatic measurements and electrostatic reticle model; and Edgar Osorio Oliveros and Vladimir Krivtsun, Maxim Spiridonov, Slava Medvedev of ISAN for the miniature ICP work. And finally, we would like to thank Pavel Krainov, Bogdan Lakatosh, and ISAN for the PIC simulations.

\section{References}

1. G. Moore, "Cramming more components onto integrated circuits," Electronics 38, 114-117 (1965).

2. W. de Boeij et al., "Enabling the $45 \mathrm{~nm}$ node by hyper NA polarized lithography," Proc. SPIE 6154, 61540B (2006).

3. E. van Setten et al., "Pushing the boundary: low-k1 extension by polarized illumination," Proc. SPIE 6520, 65200C (2007).

4. H. Jasper et al., "Immersion lithography with an ultrahigh-NA in-line catadioptric lens and a high-transmission flexible polarization illumination system," Proc. SPIE 6154, 61541W (2006).

5. R. van Es et al., "EUV for HVM: towards an industrialized scanner for HVM NXE3400B performance update," Proc. SPIE 10583, 105830H (2018).

6. M. van de Kerkhof et al., "Lithography for now and the future," Solid-State Electron. 155, 20-26 (2019).

7. D. Brouns et al., "NXE pellicle: offering a EUV pellicle solution to the industry," Proc. SPIE 9776, 97761Y (2016).

8. M. Lercel et al., "EUV reticle defectivity protection options," Proc. SPIE 11148, 111480Y (2019).

9. M. van de Kerkhof et al., "Particulate and molecular contamination control in EUV-induced H2-plasma in EUV lithographic scanner," Proc. SPIE 11489, 114890K (2020).

10. M. van de Kerkhof et al., "Understanding EUV-induced plasma and application to particle contamination control in EUV scanners," Proc. SPIE 11323, 113230Y (2020).

11. V. Bakshi, EUV Lithography, 2nd ed., SPIE Press, Bellingham, Washington (2018).

12. A. Schafgans et al., "Performance optimization of MOPA pre-pulse LPP light source," Proc. SPIE 9422, 94220B (2015).

13. M. van de Kerkhof et al., "High-power EUV lithography: spectral purity and imaging performance," J. Micro/Nanolithogr. MEMS MOEMS 19, 033801 (2020).

14. O. Braginsky et al., "Removal of amorphous $\mathrm{C}$ and $\mathrm{Sn}$ on Mo: Si multilayer mirror surface in Hydrogen plasma and afterglow," J. Appl. Phys. 111, 093304 (2012).

15. M. van der Velden et al., "Kinetic simulation of an extreme ultraviolet radiation driven plasma near a multilayer mirror," J. Appl. Phys. 100, 073303 (2006).

16. I. Mendez et al., "Atom and ion chemistry in low pressure hydrogen DC plasmas," J. Phys. Chem. A 110, 6060-6066 (2006).

17. R. Plasil et al., "Advanced integrated stationary afterglow method for experimental study of recombination of processes of $\mathrm{H}_{3+}$ and $\mathrm{H}_{3+}$ ions with electrons," Int. J. Mass Spectrom. 218, 105-130 (2002).

18. R. van der Horst et al., "Dynamics of the spatial electron density distribution of EUVinduced plasmas," J. Phys. D: Appl. Phys. 48, 432001 (2015).

19. M. van Kampen, internal ASML report (2020).

20. R. van der Horst, "Ion fluxes towards surfaces exposed to EUV-induced plasmas," Electron dynamics in EUV-induced plasmas, $\mathrm{PhD}$ thesis, University of Technology Eindhoven, Netherlands (2015).

21. T. van de Ven, "Ion fluxes towards surfaces exposed to EUV-induced plasmas," $\mathrm{PhD}$ thesis, University of Technology Eindhoven, Netherlands (2018).

22. H. Tawara et al., "Cross sections and related data for electron collisions with hydrogen molecules and molecular ions," J. Phys. Chem. Ref. Data 19(3), 617 (1990).

23. M. Lieberman, "Principles of plasma discharges and materials processing," 2nd ed., John Wiley and Sons, Inc., Hoboken, New Jersey (2005).

24. M. van de Kerkhof et al., "Advanced particle contamination control in EUV scanners," Proc. SPIE 10957, 109570U (2019). 
25. E. Louis et al., "Multilayer coated reflective optics for extreme UV lithography," Microelectron. Eng. 27, 235-238 (1995).

26. M. van der Velden et al., "Particle-in-cell Monte Carlo simulations of an extreme ultraviolet radiation driven plasma," Phys. Rev. E 73, 036406 (2006).

27. D. Astakhov, "Exploring the electron density in plasma induced by EUV radiation: II. Numerical studies in argon and hydrogen," J. Phys. D. Appl. Phys. 49, 295204 (2016).

28. T. van de Ven, "Ion energy distributions in highly transient EUV induced plasma in hydrogen," J. Appl. Phys. 123, 063301 (2018).

29. H. Kreuger, Industrial High DC Voltage: 1. Fields, 2. Breakdowns, 3. Tests, Delft University Press, Delft (1995).

30. I. Korolov et al., "Breakdown in hydrogen and deuterium gases in static and radio-frequency fields," Phys. Plasmas 22, 093501 (2015).

31. Z. Li et al., "Surface flashover of nanodielectrics with varying electrode architectures in partial vacuum under DC and kHz pulsed fields," in IPMHVC 2012 (2012).

32. M. Naidu et al., High Voltage Engineering, p. 27, McGraw-Hill (1995).

33. P. Osmokrovic et al., "Mechanism of electrical breakdown left of Paschen minimum," IEEE Trans. Dielectr. Electr. Insul. 1, 77-81 (1994).

34. E. Hastings et al., "Threshold voltage for arcing on negatively biased solar arrays," J. Spacecr. Rockets 27, 539 (1990).

35. R. Latham, High Voltage Vacuum Insulation, Author House, Bloomington (2006).

36. R. van der Wilk, ASML internal report (2018).

37. D. Cooper et al., "Electrostatic removal of particle singlets and doublets from conductive surfaces," Aerosol Sci. Technol. 12, 508-517 (1990).

38. H. Miller, "Flashover of insulators in vacuum: review of the phenomena and techniques to improved Holdoff voltage," IEEE Trans. Electr. Insul. 28, 512-527 (1993).

39. K. Asano et al., "Fundamental study of an electrostatic chuck for silicon wafer handling," Microelectron. Eng. 38, 840-845 (2002).

40. B. Oni, "Observations of voltage breakdown in ultra-low pressure environments under varied voltage and frequency conditions," J. Electr. Eng. 12, 10 (2019).

41. S. Baalrud et al., "Interaction of biased electrodes and plasmas: sheaths, double layers, and fireballs," Plasma Sources Sci. Technol. 29, 053001 (2020).

42. J. Holtzhausen et al., High Voltage Engineering Practice and Theory, Stellenbosch, Western Cape (2014).

43. J. S. Yoon et al., "Cross sections for electron collisions with hydrogen molecules," J. Phys. Chem. Ref. Data 37, 913 (2008).

44. P. McLellan, "IEDM: EUV, the road to HVM and beyond," https://community.cadence.com/ cadence_blogs_8/b/breakfast-bytes/posts/iedm-sc-euv (2019).

45. K. Ota et al., "Evaluation results of a new EUV reticle pod having reticle grounding paths," in EUVL Symp. (2010).

46. J. van Duivenbode, internal ASML report (2018).

47. A. van den Biggelaar, "Modeling of dynamic effects in high-voltage electrostatic clamps," MSc thesis, TU Eindhoven (2016).

48. G. Rider, "A critique of the approach to controlling electrostatic risk in semiconductor production and identification of a potential risk from the use of equipotential bonding," AIMS Electron. Electr. Eng. 3, 397-414 (2019).

49. M. Amemiya et al., "Experimental study of particle-free mask handling," Proc. SPIE 7271, $72713 \mathrm{G}(2009)$.

50. K. Ota et al., "Evaluation results of a new EUV reticle pod having reticle grounding paths," Proc. SPIE 7969, 79691V (2011).

51. J. Hopwood et al., "Fabrication and characterization of a micromachined $5 \mathrm{~mm}$ inductively coupled plasma generator," J. Vac. Sci. Technol. B 18, 2446 (2000).

52. E. Osorio Oliveros, internal ASML report (2019).

53. M. van de Kerkhof, "Apparatus and method," Worldwide Patent No. WO2019197128A2 (2019).

54. A. Sickafoose et al., "Experimental investigations on photoelectric and triboelectric charging of dust," J. Geophys. Res.: Space Phys. 106, 8343-8356 (2001). 
55. S. Frazier et al., "Charging behavior of dust aggregates in a cosmic plasma environment," in Lunar Planetary Sci. Conf. No. 1719 (2013).

56. H. Mott-Smith et al., "The theory of collectors in gaseous discharges," Phys. Rev. 28, 727 (1926).

57. L. Heijmans, "Quantifying plasma particle lofting," $\mathrm{PhD}$ thesis, University of Technology Eindhoven, Netherlands (2017).

58. X. Chen, "The drag force acting on a spherical non-evaporating or evaporating particle immersed into a rarefied plasma flow," J. Phys. D Appl. Phys. 29, 995 (1996).

Biographies of the authors are not available. 